\title{
Osserman manifolds of dimension 8
}

\author{
Y.Nikolayevsky*
}

\begin{abstract}
For a Riemannian manifold $M^{n}$ with the curvature tensor $R$, the Jacobi operator $R_{X}$ is defined by $R_{X} Y=R(X, Y) X$. The manifold $M^{n}$ is called pointwise Osserman if, for every $p \in M^{n}$, the eigenvalues of the Jacobi operator $R_{X}$ do not depend of a unit vector $X \in T_{p} M^{n}$, and is called globally Osserman if they do not depend of the point $p$ either. R. Osserman conjectured that globally Osserman manifolds are flat or rank-one symmetric. This Conjecture is true for manifolds of dimension $n \neq 8,16$ [14]. Here we prove the Osserman Conjecture and its pointwise version for 8-dimensional manifolds.
\end{abstract}

\section{Introduction}

An algebraic curvature tensor $R$ in a Euclidean space $\mathbb{R}^{n}$ is a $(3,1)$ tensor having the same symmetries as the curvature tensor of a Riemannian manifold. For $X \in \mathbb{R}^{n}$, the Jacobi operator $R_{X}: \mathbb{R}^{n} \rightarrow \mathbb{R}^{n}$ is defined by $R_{X} Y=R(X, Y) X$. The Jacobi operator is symmetric and $R_{X} X=0$ for all $X \in \mathbb{R}^{n}$. Throughout the paper, "eigenvalues of the Jacobi operator" refers to eigenvalues of the restriction of $R_{X}$, with $X$ a unit vector, to the subspace $X^{\perp}$.

Definition 1. An algebraic curvature tensor $R$ is called Osserman if the eigenvalues of the Jacobi operator $R_{X}$ do not depend of the choice of a unit vector $X \in \mathbb{R}^{n}$.

Definition 2. A Riemannian manifold $M^{n}$ is called pointwise Osserman if its curvature tensor is Osserman. If, in addition, the eigenvalues of the Jacobi operator are constant on $M^{n}$, the manifold $M^{n}$ is called globally Osserman.

Flat and rank-one symmetric spaces are globally Osserman, since the isometry group of each of them acts transitively on its unit tangent bundle. Osserman [16] conjectured that the converse is also true:

Osserman Conjecture. A globally Osserman manifold is flat or rank-one symmetric.

In the most cases, the answer to the Osserman Conjecture is affirmative, as well as to its "pointwise" version (see the Corollary below).

In this paper, we prove the Osserman Conjecture for 8-dimensional manifolds:

Theorem. A pointwise Osserman manifold of dimension eight is flat or rank-one symmetric.

Combining this with Theorems 1 and 2 of [14], we get:

Corollary. In each of the following cases a Riemannian manifold $M^{n}$ is flat or rank-one symmetric:

1) $M^{n}$ is globally Osserman and $n \neq 16$.

2) $M^{n}$ is pointwise Osserman and $n \neq 2,4,16$.

3) $n=16$, the manifold $M^{16}$ is (pointwise or globally) Osserman, and its Jacobi operator has no eigenvalues of multiplicity $m \in\{7,8,9\}$.

${ }^{*}$ The work is supported by ARC Discovery grant DP0342758 
In the cases covered by the Corollary, there is not much difference between globally and pointwise Osserman conditions, except in dimension 2, where any Riemannian manifold is pointwise Osserman, and in dimension 4, where any globally Osserman manifold is flat or rank-one symmetric [4], but there exist pointwise Osserman manifolds that are not symmetric ("generalized complex space forms", see [7, Corollary 2.7], [15]).

We refer to [5] for results on the Osserman Conjecture in Riemannian and semi-Riemannian geometry.

The paper is organized as follows. In Section 2, we give the proof of the Theorem, assuming two Propositions: Proposition 1 in which it is shown that all Osserman algebraic curvature tensors in $\mathbb{R}^{8}$ have a Clifford structure, and Proposition 2 which says that a Riemannian manifold with such a curvature tensor is flat or rank-one symmetric. Proposition 1 is proved in Section 3 modulo two Lemmas (their proofs are given in Section 4). Proposition 2 is proved in Section 5.

\section{Manifolds with Clifford structure. Proof of the Theorem}

We follow the two-step approach to the Osserman Conjecture suggested in [7]:

(i) find all Osserman algebraic curvature tensors;

(ii) classify Riemannian manifolds having curvature tensor as in (i).

The standard tool for (ii) is the second Bianchi identity, although the proof can sometimes be quite technically involved.

The difficult part is (i), but thanks to the remarkable construction of [6, 7], we know the right candidate for (i), a typical Osserman algebraic curvature tensor:

Definition 3. An algebraic curvature tensor $R$ in $\mathbb{R}^{n}$ has a Cliff $(\nu)$-structure $(\nu \geq 0)$, if there exist anticommuting skew-symmetric orthogonal operators $J_{1}, \ldots, J_{\nu}$, and the numbers $\lambda_{0}, \mu_{1}, \ldots \mu_{\nu}$, with $\mu_{s} \neq \lambda_{0}$, such that

$$
\begin{aligned}
R(X, Y) Z=\lambda_{0}(\langle X, Z\rangle Y- & \langle Y, Z\rangle X) \\
& +\sum_{s=1}^{\nu} \frac{1}{3}\left(\mu_{s}-\lambda_{0}\right)\left(2\left\langle J_{s} X, Y\right\rangle J_{s} Z+\left\langle J_{s} Z, Y\right\rangle J_{s} X-\left\langle J_{s} Z, X\right\rangle J_{s} Y\right) .
\end{aligned}
$$

A Riemannian manifold $M^{n}$ has a Cliff $(\nu)$-structure if its curvature tensor at every point does.

The fact that skew-symmetric operators $J_{s}$ are orthogonal and anticommute is equivalent to each of the following sets of equations: $\left\langle J_{s} X, J_{q} X\right\rangle=\delta_{s q}\|X\|^{2}$ and $J_{s} J_{q}+J_{q} J_{s}=-2 \delta_{s q} I_{n}$, for all $s, q=1, \ldots, \nu$ and all $X \in \mathbb{R}^{n}$.

The Jacobi operator of the algebraic curvature tensor $R$ with the Clifford structure given by (1) has the form

$$
R_{X} Y=\lambda_{0}\left(\|X\|^{2} Y-\langle Y, X\rangle X\right)+\sum_{s=1}^{\nu}\left(\mu_{s}-\lambda_{0}\right)\left\langle J_{s} X, Y\right\rangle J_{s} X
$$

and the tensor $R$ can be reconstructed from (2) using polarization and the first Bianchi identity.

It follows that Cliff $(\nu)$ algebraic curvature tensor (manifold) is Osserman (pointwise Osserman, respectively). Indeed, for any unit vector $X$, the Jacobi operator $R_{X}$ given by (2) has constant eigenvalues $\lambda_{0}, \lambda_{1}, \ldots, \lambda_{k-1}$, where $\lambda_{1}, \ldots, \lambda_{k-1}$ are the $\mu_{s}$ 's without repetitions. The eigenspace corresponding to the eigenvalue $\lambda_{\alpha}, \alpha \neq 0$, is $E_{\lambda_{\alpha}}(X)=\operatorname{Span}_{s: \mu_{s}=\lambda_{\alpha}}\left(J_{s} X\right)$, and the $\lambda_{0}$-eigenspace is $E_{\lambda_{0}}(X)=\left(\operatorname{Span}\left(X, J_{1} X, \ldots, J_{\nu} X\right)\right)^{\perp}$, provided $\nu<n-1$.

Following the above approach, we deduce the Theorem from two Propositions:

Proposition 1. An Osserman algebraic curvature tensor in $\mathbb{R}^{8}$ has a Clifford structure.

Proposition 2. A pointwise Osserman manifold with a Clifford structure is flat or rank-one symmetric. 


\section{Proof of Proposition 1}

Let $R$ be an Osserman algebraic curvature tensor in $\mathbb{R}^{8}$, with the Jacobi operator having $k$ distinct eigenvalues $\lambda_{0}, \lambda_{1}, \ldots, \lambda_{k-1}$ whose multiplicities are $m_{0}, m_{1}, \ldots, m_{k-1}$, respectively, with $m_{0}+m_{1}+\ldots$ $+m_{k-1}=7$. For a nonzero vector $X$, the eigenvalues of $R_{X}$ are then $0, \lambda_{0}\|X\|^{2}, \lambda_{1}\|X\|^{2}, \ldots, \lambda_{k-1}\|X\|^{2}$, and the corresponding eigenspaces are $\operatorname{Span}(X)$ and $E_{\lambda_{\alpha}\|X\|^{2}}(X)=E_{\lambda_{\alpha}}(X / \mid X \|)$, respectively. (Recall that "the eigenvalues of $R_{X}$ " are just the eigenvalues of the operator $R_{X}$ acting in $\mathbb{R}^{8}$, and there are 8 of them, counting the multiplicities. When we say "the eigenvalues of the Jacobi operator", we do not count the eigenvalue 0 corresponding to the vector $X$, so the sum of multiplicities is 7 . We allow one of the $\lambda_{\alpha}$ 's to be zero, in which case $\left.\operatorname{Ker} R_{X}=\operatorname{Span}(X) \oplus E_{0}(X)\right)$.

If $k=1$, there is nothing to prove: the curvature is constant. If $k=2$, the claim follows from [13, Proposition 1]:

Lemma 1. An Osserman algebraic curvature tensor in $\mathbb{R}^{n}, n \neq 16$, with the Jacobi operator having two distinct eigenvalues, has a Clifford structure Cliff $(\nu)$, with $\nu<n / 2$.

We may assume therefore, that $k \geq 3$. Let $m_{0}$ be the maximal multiplicity, and $\nu=7-m_{0}$ be the sum of all the others. Two cases are possible:

(A) either each of the $m_{1}, m_{2}, \ldots, m_{k-1}$ is 1 or 2 ,

(B) or $k=3$ and the multiplicities are $m_{0}=3, m_{1}=1, m_{2}=3$, up to relabelling.

To prove that the Osserman algebraic curvature tensor $R$ has a Clifford structure, it is sufficient to show that for every eigenvalue $\lambda_{\alpha}(\alpha>0)$ of the Jacobi operator, there exist $m_{\alpha}$ anticommuting skewsymmetric orthogonal operators $J_{1}^{\alpha}, \ldots, J_{m_{\alpha}}^{\alpha}$ such that for every unit vector $X$, the eigenspace $E_{\lambda_{\alpha}}(X)$ of $R_{X}$ is spanned by $J_{1}^{\alpha} X, \ldots, J_{m_{\alpha}}^{\alpha} X$. Indeed, if this is the case, then for every unit vector $X$ and every $Y \in \mathbb{R}^{n}$,

$$
R_{X} Y=\lambda_{0}\left(\|X\|^{2} Y-\langle Y, X\rangle X\right)+\sum_{\alpha=1}^{p} \sum_{s=1}^{m_{\alpha}}\left(\lambda_{\alpha}-\lambda_{0}\right)\left\langle J_{s}^{\alpha} X, Y\right\rangle J_{s}^{\alpha} X
$$

as the symmetric operators on the left-hand side and on the right-hand side have the same eigenvalues and eigenspaces. So the Jacobi operator has the form (2), up to relabelling through: $J_{1}=J_{1}^{1}, J_{2}=J_{2}^{1}$, $\ldots, J_{\nu}=J_{m_{p}}^{p}$. The only remaining thing to check is that the operators $J_{s}^{\alpha}, J_{q}^{\beta}$, with $\beta \neq \alpha$ anticommute, which easily follows from the fact that for all unit vectors $X, \quad J_{s}^{\alpha} X \in E_{\lambda_{\alpha}}(X) \perp E_{\lambda_{\beta}}(X) \ni J_{q}^{\beta} X$.

In the both cases (A) and (B) above, the proof will follow from the two Lemmas below.

First of all, as is immediate from Definition 3, shifting an algebraic curvature tensor with a Clifford structure by a constant curvature tensor results in an algebraic curvature tensor, still having a Clifford structure. So we can always assume that $\lambda_{0}$, the eigenvalue with the highest multiplicity, is zero.

Next, for all the eigenvalues of multiplicity 1 or 2 , we can find polynomial vectors spanning the corresponding eigenspaces, with the properties similar to those of the $J_{s} X$ 's:

Lemma 2. 1. Let $R$ be an Osserman algebraic curvature tensor in $\mathbb{R}^{n}$ whose Jacobi operator has $k$ distinct eigenvalues, one of which is zero, and let $\lambda \neq 0$ be a simple eigenvalue. Then for every $X \neq 0$, the eigenspace $E_{\lambda\|X\|^{2}}(X)$ of $R_{X}$ is spanned by a vector $P(X)$ all of whose components are odd homogeneous polynomial of degree $2 m+1 \leq k-1$, and for all unit vectors $X \in \mathbb{R}^{n}$,

$$
\langle P(X), X\rangle=0, \quad\|P(X)\|^{2}=1, \quad P(P(X))=-X .
$$

2. Let $R$ be an Osserman algebraic curvature tensor in $\mathbb{R}^{n}$ whose Jacobi operator has $k$ distinct eigenvalues, one of which is zero, and let $\lambda \neq 0$ be an eigenvalue of multiplicity 2 . Then for every $X \neq 0$, the eigenspace $E_{\lambda\|X\|^{2}}(X)$ of $R_{X}$ is spanned by vectors $U(X), V(X)$ all of whose components are odd homogeneous polynomial of degree $2 m+1 \leq k-1$, and for all unit vectors $X \in \mathbb{R}^{n}$,

$$
\langle U(X), X\rangle=\langle U(X), X\rangle=\langle U(X), V(X)\rangle=0, \quad\|U(X)\|^{2}=\|V(X)\|^{2}=1 .
$$


Note that in the case when the degree of $P$ (respectively, $U, V$ ) is 1 , the equations of Lemma 2 simply mean that the corresponding linear operators in $\mathbb{R}^{n}$ are orthogonal, skew-symmetric and anticommute.

The reason we require the multiplicity to be less than or equal to two is topological: any 2-dimensional plane bundle over the real projective space $\mathbb{R} P^{n}$ of dimension $n \geq 3$ is a sum of line bundles [10,8], which is no longer true for 3-dimensional bundles.

Case $A$. The Jacobi operator has three eigenvalues $0, \lambda_{1}, \lambda_{2}$ of multiplicities 3,1 , 3, respectively. From the first assertion of Lemma 2, the eigenspace $E_{\lambda_{1}\|X\|^{2}}(X)$ is spanned by a homogeneous polynomial vector $P(X)$ of degree 1 , that is, there exists an orthogonal skew-symmetric operator $J$ such that $P(X)=J X$ and $R_{X} J X=\lambda_{1}\|X\|^{2} J X$, for all $X \in \mathbb{R}^{8}$. Introduce an algebraic curvature tensor $\hat{R}$ by

$$
\hat{R}(X, Y) Z=R(X, Y) Z-\frac{\lambda_{1}}{3}(2\langle J X, Y\rangle J Z+\langle J Z, Y\rangle J X-\langle J Z, X\rangle J Y) .
$$

Its Jacobi operator has the form $\hat{R}_{X} Y=R_{X} Y-\lambda_{1}\langle J X, Y\rangle J X$. For every unit vector $X, \hat{R}_{X} J X=0$, and the restrictions of $\hat{R}_{X}$ and $R_{X}$ to $(J X)^{\perp}$ coincide, so the Jacobi operator $\hat{R}_{X}$ has two eigenvalues, $\lambda_{2}$ and 0 , with the eigenspaces $\hat{E}_{\lambda_{2}}(X)=E_{\lambda_{2}}(X)$, of dimension 3 , and $\hat{E}_{0}(X)=\operatorname{Span}(J X) \oplus E_{0}(X)$, of dimension 4 , respectively. It follows that the algebraic curvature tensor $\hat{R}$ is Osserman, with the Jacobi operator having two eigenvalues. By Lemma $1, \hat{R}$ has a Clifford structure which is a Cliff(3)-structure, and so there exist anticommuting orthogonal skew-symmetric operators $J_{1}, J_{2}, J_{3}$ in $\mathbb{R}^{8}$ such that for any unit vector $X, \hat{E}_{\lambda_{2}}(X)=\operatorname{Span}\left(J_{1} X, J_{2} X, J_{3} X\right)$.

Hence $R$ has a Clifford structure, as for any unit vector $X, E_{\lambda_{1}}(X)=\operatorname{Span}(J X)$ and $E_{\lambda_{2}}(X)=$ $\hat{E}_{\lambda_{2}}(X)=\operatorname{Span}\left(J_{1} X, J_{2} X, J_{3} X\right)$.

Case $B$. Let all the multiplicities $m_{1}, \ldots, m_{k-1}$ be 1 or 2 . From Lemma 2 it follows that for every $X \neq 0$, all the eigenspaces of the operator $R_{X}$ corresponding to the nonzero eigenvalues are spanned by polynomial vectors. Label them through $P_{1}(X), \ldots, P_{\nu}(X), \nu=m_{1}+\ldots+m_{k-1}$. The proof of the Proposition is then completed with the following Lemma (the fact that the $P_{s}(X)$ 's verify the assumptions easily follows from Lemma 2).

Lemma 3. Let $R$ be an Osserman algebraic curvature tensor in $\mathbb{R}^{8}$ whose Jacobi operator has $\nu \leq 6$ nonzero eigenvalues $\mu_{1}, \mu_{2}, \ldots, \mu_{\nu}$, counted with multiplicities. Assume that the multiplicity of each of the $\mu_{s}$ 's is not greater than $\min \{2,7-\nu\}$. Let $P_{1}(X), P_{2}(X), \ldots, P_{\nu}(X)$ be eigenvectors of $R_{X}$ such that for every $s=1, \ldots, \nu$, all the components of $P_{s}(X)$ are homogeneous polynomials of odd degree $d_{s}$, and

$$
\begin{gathered}
R_{X} P_{s}(X)=\mu_{s}\|X\|^{2} P_{s}(X), \\
\left\langle P_{s}(X), P_{t}(X)\right\rangle=\delta_{s t}\|X\|^{2 d_{s}}, \quad\left\langle P_{s}(X), X\right\rangle=0,
\end{gathered}
$$

for all $X \in \mathbb{R}^{8}$ and all $s, t=1, \ldots, \nu$, and additionally, in the case $\nu=6$,

$$
P_{s}\left(P_{s}(X)\right)=-\|X\|^{d_{s}^{2}-1} X
$$

for all $X \in \mathbb{R}^{8}$ and all $s=1, \ldots, 6$.

Then $R$ has a Cliff $(\nu)$-structure.

\section{Proof of Lemma 2 and Lemma 3}

In this Section, we give the proofs of Lemma 2 (Subsection 4.1)and Lemma 3 (Subsection 4.3). The latter one will use some algebraic results from Subsection 4.2. Throughout this Section, for $\mathbb{K}=\mathbb{R}$ or $\mathbb{C}, \mathbb{K}[X]$ is the ring of polynomials in $X=\left(x_{1}, \ldots, x_{n}\right)$ (or in $X=\left(x_{1}, \ldots, x_{8}\right)$, depending on the context) over $\mathbb{K}$. The polynomial $\|X\|^{2}=\sum_{j} x_{j}^{2}$ is irreducible in $\mathbb{K}[X]$ (for $n \geq 3$ ), and $\mathbb{K}[X]$ is a unique factorization domains (UFD). We will say that a matrix (a vector) with entries from $\mathbb{K}[X]$ is divisible by $\|X\|^{2}$, if each of its entries is. 


\subsection{Proof of Lemma 2}

The first assertion of Lemma 2 follows from Lemma 2.1 of [12]. Here we prove the second one. Throughout the proof, we will freely switch between operators and their matrices using the same notation. We call a finite set of polynomials coprime, if their greatest common divisor is one.

Let $\lambda_{0}=0, \lambda_{1}=\lambda, \lambda_{2}, \ldots, \lambda_{k-1}$ be the eigenvalues of the Jacobi operator.

For every $X \in \mathbb{R}^{n}$, define a symmetric operator $W_{X}: \mathbb{R}^{n} \rightarrow \mathbb{R}^{n}$ by $W_{X}=\rho^{-1} R_{X} \prod_{\alpha=2}^{k-1}\left(R_{X}-\right.$ $\left.\lambda_{\alpha}\|X\|^{2} I_{n}\right)$, where $\rho=\lambda \prod_{\alpha=2}^{k-1}\left(\lambda-\lambda_{\alpha}\right)$. The operator $W_{X}$ is an orthogonal projection on the 2plane $E_{\lambda\|X\|^{2}}(X)$ followed by multiplication by $\|X\|^{2 k-2}$, and the entries of its matrix are homogeneous polynomials of even degree $2 k-2$ of the coordinates $x_{1}, \ldots, x_{n}$ of vector $X$, so that

$$
W_{X}^{2}=\|X\|^{2 k-2} W_{X}, \quad \text { and } \operatorname{rk} W_{X}=2 \text { when } \quad X \neq 0 .
$$

Let $q(X)$ be the greatest common divisor of the entries of the matrix $W_{X}$ over $\mathbb{R}[X]$. Then $q(X)$ divides $\operatorname{Tr} W_{X}=2\|X\|^{2 k-2}$, and so $q(X)=\|X\|^{2 k-2-2 s}$ for some $s \leq k-1$. Dividing $W_{X}$ by $q(X)$ we get a symmetric polynomial matrix $S_{X}$ whose entries are coprime homogeneous polynomials of degree $2 s$ satisfying

$$
S_{X}^{2}=\|X\|^{2 s} S_{X}, \quad \quad \operatorname{rk} S_{X}=2 \quad \text { when } \quad X \neq 0,
$$

and the two-dimensional $\|X\|^{2 s}$-eigenspace of $S_{X}$ is $E_{\lambda\|X\|^{2}}(X)$.

Since for any unit vector $X, E_{\lambda}(X)=E_{\lambda}(-X)$, the two-plane bundle $E_{\lambda}$ over the unit sphere in $\mathbb{R}^{n}$ descends to a two-plane bundle over $\mathbb{R} P^{n-1}$. Any such bundle is a sum of two line bundles when $n \geq 3$ $[10,8]$, so we can choose two orthonormal vector fields $s_{1}(X), s_{2}(X)$ spanning $E_{\lambda}(X)$ for $X \in S^{n-1}$ which are odd with respect to the antipodal map.

Let $\tilde{s}_{1}, \tilde{s}_{2}$ be vector fields in $\mathbb{R}^{n}$ whose components are odd homogeneous polynomials of the same degree and whose restrictions to $S^{n-1}$ are uniformly close to $s_{1}, s_{2}$, respectively. As the polynomial matrix $S_{X}$ is a projection on the 2-plane $E_{\lambda}(X /\|X\|)$ followed by multiplication by $\|X\|^{2 s}$, the components of vector fields

$$
P_{1}(X)=S_{X} \tilde{s}_{1}(X), \quad P_{2}(X)=S_{X} \tilde{s}_{2}(X)
$$

are odd homogeneous polynomials of the same degree, with $P_{1}(X)$ and $P_{2}(X)$ pointwise linearly independent on the sphere $S^{n-1}$ (and hence in $\mathbb{R}^{n} \backslash 0$ ), and spanning $E_{\lambda\|X\|^{2}}(X)$. Replacing $P_{1}$ by $\left\|P_{2}\right\|^{2} P_{1}-$ $\left\langle P_{1}, P_{2}\right\rangle P_{2}$, we can assume that for all $X, P_{1}(X) \perp P_{2}(X)$. Dividing each of $P_{1}(X), P_{2}(X)$ by the greatest common divisor of its components, we get two homogeneous polynomial vectors $P_{1}(X), P_{2}(X)$, each with coprime components, and such that

$$
\left\langle P_{1}(X), X\right\rangle=\left\langle P_{2}(X), X\right\rangle=\left\langle P_{1}(X), P_{2}(X)\right\rangle=0, \quad S_{X} P_{i}(X)=\|X\|^{2 s} P_{i}(X),
$$

for all $X \in \mathbb{R}^{n}, i=1,2$. The polynomials $a(X)=\left\|P_{1}(X)\right\|^{2}$ and $b(X)=\left\|P_{2}(X)\right\|^{2}$ are nonzero outside the origin in $\mathbb{R}^{n}$, and both $P_{1}$ and $P_{2}$ are still odd, as from $P_{1}(X), P_{2}(X) \perp X$ it follows that the self-maps of $S^{n-1}$ defined by $X \rightarrow a(X)^{-1 / 2} P_{1}(X), X \rightarrow b(X)^{-1 / 2} P_{2}(X)$ are homotopic to the identity.

From (6) and (7), we get the following matrix equation:

$$
\left(P_{1} \mid P_{2}\right)\left(\begin{array}{cc}
a^{-1} & 0 \\
0 & b^{-1}
\end{array}\right)\left(P_{1} \mid P_{2}\right)^{t}=\|X\|^{-2 s} S_{X}
$$

for all $X \neq 0$. Let $a=a^{\prime} c, b=b^{\prime} c$, with $a^{\prime}, b^{\prime}$ coprime polynomials. Then

$$
\|X\|^{2 s} b^{\prime}(X) P_{1}(X) P_{1}(X)^{t}+\|X\|^{2 s} a^{\prime}(X) P_{2}(X) P_{2}(X)^{t}=a^{\prime}(X) b^{\prime}(X) c(X) S_{X},
$$

so the polynomial matrix $\|X\|^{2 s} b^{\prime}(X) P_{1}(X) P_{1}(X)^{t}$ is divisible by $a^{\prime}(X)$. Since $a^{\prime}$ and $b^{\prime}$ are coprime and the components of $P_{1}(X)$ are coprime, $\|X\|^{2 s}$ must be divisible by $a^{\prime}(X)$, so that $a^{\prime}(X)=\|X\|^{2 m}$, 
for some $m \leq s$. Similarly, $b^{\prime}(X)=\|X\|^{2 m^{\prime}}$. Again, since $a^{\prime}$ and $b^{\prime}$ are coprime, no more than one of the numbers $m, m^{\prime}$ can be nonzero. Let say $m^{\prime}=0$, that is, $b^{\prime}(X)=1$. Then

$$
\|X\|^{2 s-2 m} P_{1}(X) P_{1}(X)^{t}+\|X\|^{2 s} P_{2}(X) P_{2}(X)^{t}=c(X) S_{X} .
$$

Since the polynomial $\|X\|^{2}$ is irreducible, and the entries of $S_{X}$ are coprime, $c(X)=\|X\|^{2 s-2 m} d(X)$ for some polynomial $d$, and we come to

$$
P_{1}(X) P_{1}(X)^{t}+\|X\|^{2 m} P_{2}(X) P_{2}(X)^{t}=d(X) S_{X} .
$$

The number $m$ must be even. Indeed, both $P_{1}(X)$ and $P_{2}(X)$ are of odd degree, so the polynomials $a(X)=\left\|P_{1}(X)\right\|^{2}$ and $b(X)=\left\|P_{2}(X)\right\|^{2}$ are of degree $2 \bmod 4$. But $a=a^{\prime} c=\|X\|^{2 s} d(X), b=c=$ $\|X\|^{2 s-2 m} d(X)$, so $2 m$ is divisible by 4 .

Then $\|X\|^{m}$ is a polynomial and we can introduce polynomial vectors $P=P_{1}, Q=\|X\|^{m} P_{2}$ which satisfy $P(X) P(X)^{t}+Q(X) Q(X)^{t}=d(X) S_{X}$, and so for every $Y \in \mathbb{R}^{n}$,

$$
\langle P(X), Y\rangle^{2}+\langle Q(X), Y\rangle^{2}=d(X)\left\langle S_{X} Y, Y\right\rangle .
$$

The components of $P(X)$ are still coprime over $\mathbb{R}[X]$, while the greatest common divisor of the components of $Q(X)$ is $\|X\|^{m}$. We want to find two polynomial vectors $U(X), V(X)$ spanning the same subspace as $P(X), Q(X)$ (which is the eigenspace $E_{\lambda\|X\|^{2}}(X)$ of $\left.R_{X}\right)$ and satisfying (8) with $d(X)=1$.

Let $r(Z)$ be an irreducible factor of $d(Z)$ over $\mathbb{C}[Z]$. Then either $r(Z)$ is essentially real, that is, $\bar{r}(Z)=r(Z)$ up to multiplication by a nonzero complex number, or $d(Z)$ is also divisible by $\bar{r}(Z)$ (where $\bar{f}$ is defined by $\bar{f}(Z)=\overline{f(\bar{Z})})$. In both cases, (8) implies that for every $Y \in \mathbb{R}^{n}$, the complex polynomial $\langle P(Z)+i Q(Z), Y\rangle\langle P(Z)-i Q(Z), Y\rangle$ is divisible by $r(Z)$ over $\mathbb{C}[Z]$ (the scalar product is Euclidean, not Hermitian). Let say $\langle P(Z)+i Q(Z), Y\rangle$ be divisible by $r(Z)$, for all real $Y$, so that $r(Z)$ divides every component of the vector $P(Z)+i Q(Z)$.

In the first case, since $\bar{P}=P, \bar{Q}=Q, \bar{r}=r$, the polynomial $r(Z)$ also divides every component of $P(Z)-i Q(Z)$. Then every component of $P(Z)$ is divisible by $r(Z)$ over $\mathbb{C}[Z]$, and so every component of $P(X)$ is divisible by $r(X)$ over $\mathbb{R}[X]$. This contradicts to the fact that the components of $P$ are coprime over $\mathbb{R}[X]$.

In the second case, let $F(Z)$ be a polynomial vector such that $P(Z)+i Q(Z)=r(Z) F(Z)$. For real $Z=X$, let $r(X)=r_{1}(X)+i r_{2}(X), F(X)=A(X)+i B(X)$, with $r_{1}, r_{2}$ real polynomials, and $A, B$ real polynomial vectors. Then $A=\left(P r_{1}+Q r_{2}\right)\left(r_{1}^{2}+r_{2}^{2}\right)^{-1}, B=\left(-P r_{2}+Q r_{1}\right)\left(r_{1}^{2}+r_{2}^{2}\right)^{-1}$, and so

$$
\begin{aligned}
A(X) A(X)^{t}+B(X) B(X)^{t}=\left(r_{1}(X)^{2}+r_{2}(X)^{2}\right)^{-1}\left(P(X) P(X)^{t}\right. & \left.+Q(X) Q(X)^{t}\right) \\
& =\left(\left(r_{1}(X)^{2}+r_{2}(X)^{2}\right)^{-1} d(X)\right) S_{X} .
\end{aligned}
$$

Since $d(Z)$ is divisible by irreducible polynomials $r(Z)$ and $\bar{r}(Z)$ over $\mathbb{C}[Z]$, it is also divisible by $r(Z) \bar{r}(Z)$. Then, over $\mathbb{R}[X]$, the real polynomial $d(X)$ is divisible by the real polynomial $r(X) \bar{r}(X)=r_{1}(X)^{2}+$ $r_{2}(X)^{2}$. Hence $\left(r_{1}(X)^{2}+r_{2}(X)^{2}\right)^{-1} d(X)$ is a polynomial, of degree less than $\operatorname{deg} d(X)$.

Repeating this procedure, we finally come to two polynomial vectors, $U(X)$ and $V(X)$, such that

$$
U(X) U(X)^{t}+V(X) V(X)^{t}=S_{X} .
$$

It remains to check that $U(X), V(X)$ is the sought pair of polynomial vectors.

Since by $(6) \operatorname{rk} S_{X}=2$ for all $X \neq 0$, the vectors $U(X), V(X)$ must be pointwise linearly independent outside the origin. Substituting $S_{X}$ from (9) to (6) we get $U(X)\left(\left(\|U(X)\|^{2}-\|X\|^{2 s}\right) U(X)^{t}+\right.$ $\left.\langle U(X), V(X)\rangle V(X)^{t}\right)+V(X)\left(\left(\|V(X)\|^{2}-\|X\|^{2 s}\right) V(X)^{t}+\langle U(X), V(X)\rangle U(X)^{t}\right)=0$ which implies, by the linear independence of $U(X)$ and $V(X)$, that

$$
\|U(X)\|^{2}=\|V(X)\|^{2}=\|X\|^{2 s}, \quad\langle U(X), V(X)\rangle=0 .
$$


It also follows from (9), that all the components of $U(X)$ and $V(X)$ are homogeneous polynomials of degree $s$, and for $X \neq 0$, the vectors $U(X), V(X)$ span $E_{\lambda\|X\|^{2}}(X)$. In particular, as $E_{\lambda\|X\|^{2}}(X) \perp X$,

$$
\langle U(X), X\rangle=\langle V(X), X\rangle=0
$$

and so $s=\operatorname{deg} U(X)=\operatorname{deg} V(X)$ is odd (otherwise the homotopy $\cos \theta X+\sin \theta U(X)$ joins the identity map of $S^{n-1}$ with the one of even topological degree). Finally, by construction of $S_{X}, s \leq k-1$.

\subsection{Two algebraic facts}

Let for $X=\left(x_{1}, \ldots, x_{8}\right), \quad\left(\|X\|^{2}\right)$ be the ideal of $\mathbb{R}[X]$ generated by $\|X\|^{2}=\sum_{j} x_{j}^{2}$, and $\mathbf{R}=$ $\mathbb{R}[X] /\left(\|X\|^{2}\right)$, with $\pi: \mathbb{R}[X] \rightarrow \mathbf{R}$ the natural projection. The field of fractions $\mathbb{F}$ of the ring $\mathbf{R}$ is isomorphic to the field $\mathbb{L}_{7}=\mathbb{R}\left(x_{1}, \ldots, x_{7}, \sqrt{-d}\right.$ ), where $d=x_{1}^{2}+\ldots+x_{7}^{2}$ (an isomorphism from $\mathbb{L}_{7}$ to $\mathbb{F}$ is induced by the map $(a+b \sqrt{-d}) / c \rightarrow\left(a+b x_{8}\right) / c$, with $\left.a, b, c \in \mathbb{R}\left[x_{1}, \ldots, x_{7}\right], c \neq 0\right)$.

The level $s(\mathbb{k})$ of a field $\mathbb{k}$ is the minimal number of elements $c_{1}, \ldots, c_{m} \in \mathbb{k}$ such that $c_{1}^{2}+c_{2}^{2}+\ldots+$ $c_{m}^{2}=-1$ [17]. If -1 is not a sum of squares in $\mathbb{k}$, then $s(\mathbb{k})=\infty$. By Pfister's Theorem, the level of any field is a power of 2 , if finite.

We need the following two facts:

Fact 1 ([11]). The rings $\mathbf{R}$ and $\mathbf{R} \otimes \mathbb{C}=\mathbb{C}[X] /\left(\|X\|^{2}\right)$ are unique factorization domains (UFD's).

Fact 2 ([17, Theorem 3.1.4]). The level of the field $\mathbb{F}\left(\cong \mathbb{L}_{7}\right)$ is 4 .

Remark. Fact 2 is equivalent to the following: if a sum of squares of no more than four elements in $\mathbf{R}$ is zero, than all the elements are zeros. Indeed, if $a_{1}^{2}+\ldots+a_{m}^{2}=0$ for $a_{1}, \ldots, a_{m} \in \mathbf{R}$, with $m \leq 4$, and say $a_{m} \neq 0$, then in $\mathbb{F}, \quad\left(a_{1} / a_{m}\right)^{2}+\ldots+\left(a_{m-1} / a_{m}\right)^{2}=-1$ which is a contradiction, as $m-1<4$.

Equivalently, if a sum of squares of no more than four real polynomials in eight variables $x_{1}, \ldots, x_{8}$ is divisible by $\|X\|^{2}$, then each of them is. In the proof of Proposition 2 (Lemma 6), we will also use a similar fact for polynomials in six variables: if a sum of squares of two real polynomials in $x_{1}, \ldots, x_{6}$ is divisible by $\sum_{i=1}^{6} x_{i}^{2}$, then both are divisible by $\sum_{i=1}^{6} x_{i}^{2}$ (this also follows from [17, Theorem 3.1.4]: the level of the field $\mathbb{L}_{5}=\mathbb{R}\left(x_{1}, \ldots, x_{5}, \sqrt{-\left(x_{1}^{2}+\ldots+x_{5}^{2}\right)}\right)$ is 4$)$.

In the proof of Lemma 3, we will use the following Lemma for polynomial matrices.

Lemma 4. Let $A$ be an $8 \times m$ polynomial matrix (with entries in $\mathbb{R}[X]$ ), and $D$ be a real diagonal $m \times m$ matrix with positive diagonal entries. Assume that $A D A^{t}$ is divisible by $\|X\|^{2}$. Then:

(i) If $m \leq 4$, then $A$ itself is divisible by $\|X\|^{2}$.

(ii) If $m=5$, then there exist polynomial vectors $U$ and $Q$ of dimension 8 and 5 , respectively, and an $8 \times 5$ polynomial matrix $B$ such that

$$
A=U Q^{t}+\|X\|^{2} B, \quad \text { and the polynomial } Q^{t} D Q=\langle D Q, Q\rangle \text { is divisible by }\|X\|^{2} .
$$

Proof. Denote $d_{1}, \ldots, d_{m}>0$ the diagonal entries of the matrix $D$. Let $a$ be an $8 \times m$ matrix with entries in $\mathbf{R}$, such that $a_{j s}=\pi\left(\sqrt{d_{s}} A_{j s}\right), s=1, \ldots, m, j=1, \ldots, 8$. Then by assumption, $a a^{t}=0$ which implies that for any two rows $a_{i}, a_{j}$ of the matrix $a, \quad\left\|a_{i}\right\|^{2}=\left\|a_{j}\right\|^{2}=\left\langle a_{i}, a_{j}\right\rangle=0$ (where $\langle\cdot, \cdot\rangle$ and $\|\cdot\|^{2}$ in the free module $\mathbf{R}^{m}$ have an obvious meaning).

(i) If $m \leq 4$, then, for every $j,\left\|a_{j}\right\|^{2}$ is a sum of no more than four squares in $\mathbf{R}$, and so the equation $\left\|a_{j}\right\|^{2}=0$ implies $a_{j}=0$ by Fact 2 .

(ii) Let $m=5$. We have to find $u \in \mathbf{R}^{8}, q \in \mathbf{R}^{5}$ such that $a=u q^{t}$ (then automatically $\|q\|^{2}=0$, unless $a=0$, in which case there is nothing to prove). 
Without loss of generality, assume that $a_{11} \neq 0$. Denote $u_{1}$ the greatest common divisor of the entries in the first row of $a(\mathbf{R}$ is a UFD by Fact 1$)$, and let $a_{1 s}=u_{1} q_{s}, s=1, \ldots, 5$, with the $q_{s}$ 's coprime (note that none of the $q_{s}$ 's is zero, because otherwise $0=\left\|a_{1}\right\|^{2}$ is a sum of no more than four squares, and so $\left.a_{1}=0\right)$.

As for any two rows $a_{i}, a_{j} \quad\left\|a_{i}\right\|^{2}=\left\|a_{j}\right\|^{2}=\left\langle a_{i}, a_{j}\right\rangle=0$, it follows that for all $j=2, \ldots, 8, \quad \| a_{11} a_{j}-$ $a_{j 1} a_{1} \|^{2}=0$ which is a sum of no more than four squares in $\mathbf{R}$. So by Fact $2, a_{11} a_{j}=a_{j 1} a_{1}$, that is, $q_{1} a_{j s}=a_{j 1} q_{s}$ for all $j \geq 2, s$. As $\mathbf{R}$ is a UFD, there exist $u_{2}, \ldots, u_{8}$ such that $a_{j s}=u_{j} q_{s}$, for all $j, s$.

\subsection{Proof of Lemma 3}

Without loss of generality, we can assume that none of the polynomial vectors $P_{s}(X)$ is divisible by $\|X\|^{2}$ (for every $s$, at least one of the components of $P_{s}(X)$ is not divisible by $\|X\|^{2}$ ).

Let $d=\max \left\{d_{1}, \ldots, d_{\nu}\right\}$. Multiplying the vectors $P_{s}(X)$, with $d_{s}<d$, by an appropriate power of $\|X\|^{2}$, we can assume that all the components of all the $P_{s}$ 's have degree $d$, and at least one of the $P_{s}(X)$ 's is still not divisible by $\|X\|^{2}$.

We want to show that $d=1$, that is, each $P_{s}$ is a linear operator in $\mathbb{R}^{8}$. Assume that $d>1$ (recall that all the $d_{s}$ 's are odd, and so is $d$ ).

Let $A(X)$ be $8 \times \nu$ polynomial matrix whose columns are $P_{1}(X), \ldots, P_{\nu}(X)$, and let $\Lambda$ be $\nu \times \nu$ constant diagonal matrix with diagonal entries $\mu_{1}, \ldots, \mu_{\nu}$. Then by $(3)$ the symmetric $8 \times 8$ matrices $A(X) \Lambda A(X)^{t}$ and $\|X\|^{2 d-2} R_{X}$ have the same eigenspaces and eigenvalues for all $X \neq 0$, so

$$
A(X) \Lambda A(X)^{t}=\|X\|^{2 d-2} R_{X}, \quad A(X)^{t} A(X)=\|X\|^{2 d} I_{8},
$$

(the second equation follows from (4)). Raising both sides of the first equation of (11) to the power $k<d$ and using the second one we get

$$
A(X) \Lambda^{k} A(X)^{t}=\|X\|^{2(d-k)} R_{X}^{k}, \quad k=1, \ldots, d-1,
$$

so all the polynomial matrices $A(X) \Lambda^{k} A(X)^{t}$ with $k \leq d-1$ are divisible by $\|X\|^{2}$. We are going to prove that the matrix $A(X)$ is itself divisible by $\|X\|^{2}$. This will lead to a contradiction with the fact that at least one of the $P_{s}(X)$ 's is not divisible by $\|X\|^{2}$.

Let $\nu \leq 4$. By (12), the matrix $A(X) \Lambda^{2} A(X)^{t}$ is divisible by $\|X\|^{2}$, with $\Lambda^{2}$ diagonal, positive definite matrix. Then by Lemma $4(\mathrm{i}), A(X)$ is divisible by $\|X\|^{2}$.

Let $\nu=5$. Without loss of generality, assume that the $\mu_{s}$ 's are labelled in such a way that $\mu_{1}^{-1} \leq$ $\mu_{2}^{-1} \leq \ldots \leq \mu_{5}^{-1}$. Let $m(=1$ or 2$)$ be the multiplicity of $\mu_{1}$, and let $A(X)=\left(A_{1} \mid A_{2}\right)$, where $A_{1}, A_{2}$ are submatrices of $A(X)$ consisting of the first $m$, and the last $5-m$ columns, respectively. Denote $\widetilde{\Lambda}$ the $(5-m) \times(5-m)$ diagonal matrix whose diagonal entries are $\mu_{m+1}-\mu_{1}, \ldots, \mu_{5}-\mu_{1}$. Then from (11) and (12), with $k=2$, both matrices

$$
\mu_{1} A_{1} A_{1}^{t}+A_{2} \widetilde{\Lambda} A_{2}^{t}, \quad \text { and } \quad \mu_{1}^{2} A_{1} A_{1}^{t}+A_{2} \widetilde{\Lambda}^{2} A_{2}^{t} \quad \text { are divisible by }\|X\|^{2},
$$

and then, so is $A_{2}\left(\widetilde{\Lambda}^{2}-\mu_{1} \widetilde{\Lambda}\right) A_{2}^{t}$. By Lemma 4(i), this implies that $A_{2}$ is divisible by $\|X\|^{2}$, as $5-m \leq 4$ and the diagonal matrix $\widetilde{\Lambda}^{2}-\mu_{1} \widetilde{\Lambda}$ is definite: all its diagonal entries $\mu_{s}^{2}-\mu_{1} \mu_{s}=\mu_{s}^{2} \mu_{1}\left(\mu_{1}^{-1}-\mu_{s}^{-1}\right)$ have the same sign. Then $\mu_{1} A_{1} A_{1}^{t}$ is divisible by $\|X\|^{2}$ by (13), and so is $A_{1}$, again by Lemma 4(i).

Let now $\nu=6$. In this case, by the assumption, all the $\mu_{s}$ 's are distinct. We can assume that they are labelled in such a way that $\mu_{1}^{-1}<\mu_{2}^{-1}<\ldots<\mu_{6}^{-1}$.

First consider the case $d \geq 5$. Then by (12), all four polynomial matrices $A(X) \Lambda^{k} A(X)^{t}, k=1,2,3,4$ are divisible by $\|X\|^{2}$. Set $f(t)=t\left(t-\mu_{1}\right)\left(t-\mu_{2}\right)\left(t-\mu_{3}\right)$, and denote $\widetilde{\Lambda}=\operatorname{diag}\left\{f\left(\mu_{4}\right), f\left(\mu_{5}\right), f\left(\mu_{6}\right)\right\}$. Note that all three diagonal entries $f\left(\mu_{4}\right), f\left(\mu_{5}\right), f\left(\mu_{6}\right)$ of $\widetilde{\Lambda}$ have the same sign. Let $A(X)=\left(A_{1} \mid A_{2}\right)$, where $A_{1}$ and $A_{2}$ consist of the first three and the last three columns of $A(X)$, respectively. Then the 
matrix $A(X) f(\Lambda) A(X)^{t}=A_{2} \widetilde{\Lambda} A_{2}^{t}$ is divisible by $\|X\|^{2}$, as $f(\Lambda)$ is a linear combination of the $\Lambda^{k}$,s, $k=1,2,3,4$. Lemma $4(\mathrm{i})$ implies that $A_{2}$ is divisible by $\|X\|^{2}$. Then by (12) with $k=2$, the matrix $A_{1} \operatorname{diag}\left\{\mu_{4}^{2}, \mu_{5}^{2}, \mu_{6}^{2}\right\} A_{1}^{t}$ is also divisible by $\|X\|^{2}$, and so $\|X\|^{2}$ divides $A_{1}$, as well.

Let now $d=3$, so that all the entries of $A(X)$ are homogeneous cubic polynomials. Let $\hat{A}$ be an $8 \times 5$ matrix obtained from $A(X)$ by crossing out the last column. We can assume that $\hat{A}$ is not divisible by $\|X\|^{2}$, as otherwise from (11) it follows that $A(X)$ is also divisible by $\|X\|^{2}$.

As $\mu_{1}^{-1}<\mu_{2}^{-1}<\ldots<\mu_{6}^{-1}$, all the numbers $\alpha_{s}=\mu_{s}^{2}-\mu_{s} \mu_{6}$ with $s \leq 5$ have the same sign. So a $5 \times 5$ matrix $\tilde{\Lambda}=\operatorname{diag}\left\{\alpha_{1}, \ldots, \alpha_{5}\right\}$ is definite. By (12), the polynomial matrix $A(X)\left(\Lambda^{2}-\mu_{1} \Lambda\right) A(X)^{t}=\hat{A} \tilde{\Lambda} \hat{A}^{t}$ is divisible by $\|X\|^{2}$. Then by Lemma 4(ii), we can find polynomial vectors $U$ and $Q$ of dimension 8 and 5 , respectively, and a polynomial matrix $B$, such that

$$
\begin{gathered}
\hat{A}=U Q^{t}+\|X\|^{2} B, \quad \text { and } \\
\sum_{s=1}^{5} \alpha_{s} q_{s}^{2} \quad \text { is divisible by }\|X\|^{2},
\end{gathered}
$$

where $Q^{t}=\left(q_{1}, \ldots, q_{5}\right)$. Note that none of the $q_{s}$ 's is divisible by $\|X\|^{2}$, because otherwise by Lemma 4(i), the equation (15) implies that all of them are, and so $\hat{A}$ is divisible by $\|X\|^{2}$. Moreover, from (11),

$$
\hat{A}^{t} \hat{A}=\|X\|^{6} I_{8}
$$

and, in particular,

$$
\sum_{j=1}^{8} u_{s}^{2} \text { is divisible by }\|X\|^{2}
$$

We can choose, for every $j$ and $s$, the components $u_{j}, q_{s}$ of $U, Q$ to be of the lowest possible degree in the cosets $u_{j}+\left(\|X\|^{2}\right)$ and $q_{s}+\left(\|X\|^{2}\right)$, respectively. Then for all $s, q_{s} \neq 0$; some of the $u_{j}$ 's can vanish, but not all of them, as otherwise $\hat{A}$ is divisible by $\|X\|^{2}$.

Let $l_{s}=\operatorname{deg} q_{s}$ and $r_{j}=\operatorname{deg} u_{j}$ for those $j$ for which $u_{j} \neq 0$. Then $l_{s}+r_{j} \leq 3$, because otherwise from (14) it will follow that the product of the highest degree terms of $q_{s}$ and $u_{j}$ is divisible by $\|X\|^{2}$, and so we can decrease the degree of one of them. Also from (14), $l_{s}+r_{j} \geq 2$, as the entries of the matrix $\hat{A}-\|X\|^{2} B$ have no terms of degree lower than 2 . If for some $s, j, l_{s}+r_{j}=2$, then by (14) $q_{s} u_{j}=(\hat{A})_{j s}-\|X\|^{2} B_{j s}$, and so $q_{s} u_{j}$ is divisible by $\|X\|^{2}$, as $\operatorname{deg}(\hat{A})_{j s}=3$. Then $u_{j}$ is divisible by $\|X\|^{2}$ (as $q_{s}$ is not) which contradicts to the choice of $u_{j}$.

So for all $s$ and all $j$ with $u_{j} \neq 0, l_{s}+r_{j}=3$, hence $l_{s}=l, r_{j}=r$, and $l+r=3$. Note that by (15), $r \geq 2$, as a sum of squares of less than eight nonzero linear polynomials cannot be divisible by $\|X\|^{2}$. Similarly, by (17), $l \geq 1$, and if $l=1$, then all the $u_{j}$ 's are non-zero. So, for all $s, j, \operatorname{deg} q_{s}=2, \operatorname{deg} u_{j}=1$. As $q_{s} u_{j}=(\hat{A})_{j s}-\|X\|^{2} B_{j s}$, by (14), none of the $q_{s}, u_{j}$ contain lower degree terms, that is, $q_{s}, u_{j}$ are homogeneous.

So there exists an $8 \times 8$ real matrix $V$ such that $U=V X$. It follows immediately from (17), that $V$ is proportional to an orthogonal matrix: $V V^{t}=\beta I_{8}, \beta>0$ (if $\beta=0$, then $U=0$, and so $\hat{A}$ is divisible by $\left.\|X\|^{2}\right)$.

Consider the polynomial vector $P_{1}(X)$, the first column of the matrix $\hat{A}$. We have

$$
P_{1}(X)=\left\langle M_{1} X, X\right\rangle V X+\|X\|^{2} B_{1}(X),
$$

where $M_{1}$ is the matrix of the quadratic form $q_{1}$ and $B_{1}(X)$ is the first column of the matrix $B(X)$. Then the equation (5) implies

$$
-X\|X\|^{8}=P_{1}\left(P_{1}(X)\right)=\left\langle M_{1} P_{1}(X), P_{1}(X)\right\rangle V P_{1}(X)+\left\|P_{1}(X)\right\|^{2} B_{1}\left(P_{1}(X)\right) .
$$

But $\left\|P_{1}(X)\right\|^{2}=\|X\|^{6}$ by (4), so the vector $\left\langle M_{1} P_{1}(X), P_{1}(X)\right\rangle V P_{1}(X)$ must be divisible by $\|X\|^{2}$. Substituting $P_{1}(X)$ from (18) we find that $\|X\|^{2}$ divides $\left\langle M_{1} X, X\right\rangle^{3}\left\langle M_{1} V X, V X\right\rangle V^{2} X$. Since the polynomial 
$\left\langle M_{1} X, X\right\rangle\left(=q_{1}\right)$ is not divisible by $\|X\|^{2}$, the vector $\left\langle M_{1} V X, V X\right\rangle V^{2} X$ must be divisible by $\|X\|^{2}$. As $V$ is a nonzero multiple of an orthogonal matrix, the matrix $V^{2}$ is nonzero, and so $\left\langle M_{1} V X, V X\right\rangle=\gamma\|X\|^{2}$ for some $\gamma \in \mathbb{R}$. Then $V^{t} M_{1} V=\gamma I_{8}$, so $M_{1}=\gamma \beta^{-1} I_{8}$, and $q_{1}=\left\langle M_{1} X, X\right\rangle=\gamma \beta^{-1}\|X\|^{2}$ which is a contradiction.

Thus, all the entries of the matrix $A(X)=\left(P_{1}(X)|\ldots| P_{\nu}(X)\right)$ are linear in $X$, so there exist linear operators $J_{s}$ in $\mathbb{R}^{8}$ such that $P_{s}(X)=J_{s} X, s=1, \ldots, \nu$. It easily follows from (4) that $J_{s}$ 's are orthogonal, skew-symmetric, and anticommute. Also, by $(11)$, for any $X, Y \in \mathbb{R}^{8}$,

$$
R_{X} Y=A(X) \Lambda A(X)^{t} Y=\left(J_{1} X|\ldots| J_{\nu} X\right) \operatorname{diag}\left\{\mu_{1}, \ldots, \mu_{\nu}\right\}\left(J_{1} X|\ldots| J_{\nu} X\right)^{t} Y=\sum_{s=1}^{\nu} \mu_{s}\left\langle J_{s} X, Y\right\rangle J_{s} X
$$

hence the algebraic curvature tensor $R$ has a Cliff $(\nu)$-structure.

\section{Proof of Proposition 2}

Let the curvature tensor $R$ of the manifold $M^{8}$ have a Clifford structure Cliff $(\nu)$, that is, there exist $\nu$ fields of anticommuting skew-symmetric orthogonal operators $J_{1}, \ldots, J_{\nu}$, and the functions $\lambda_{0}, \lambda_{1}, \ldots, \lambda_{\nu}\left(\lambda_{i} \neq \lambda_{0}\right.$ for $\left.i>0\right)$ such that for any three vectors $X, Y, Z$,

$$
\begin{aligned}
R(X, Y) Z=\lambda_{0}(\langle X, Z\rangle Y-\langle Y, Z\rangle X) & \\
& +\sum_{i=1}^{\nu} \frac{1}{3}\left(\lambda_{i}-\lambda_{0}\right)\left(2\left\langle J_{i} X, Y\right\rangle J_{i} Z+\left\langle J_{i} Z, Y\right\rangle J_{i} X-\left\langle J_{i} Z, X\right\rangle J_{i} Y\right)
\end{aligned}
$$

We work in a neighbourhood $\mathcal{U}$ of a generic point on $M^{8}$, so that we assume both $\nu$ and the number of pairwise nonequal $\lambda_{i}$ 's to be locally constant. As $M^{8}$ is Einstein, and hence analytic, it is sufficient to prove the Proposition for $\mathcal{U}$ only.

For $\nu \leq 2$ the proof follows from the results of $[7,12]$, so we will assume $3 \leq \nu \leq 7$. Also, by the result of [13], we can assume that the Jacobi operator has at least three different eigenvalues.

To avoid considering too many cases, we start with the following Lemma.

Lemma 5. One of two possibilities can occur:

(a) There exist $7-\nu$ skew-symmetric orthogonal operators $J_{\nu+1}, \ldots, J_{7}$ such the operators $J_{1}, \ldots, J_{7}$ anticommute, $J_{1} J_{2} \ldots J_{7}=I_{8}$, and the curvature tensor has the form

$$
R(X, Y) Z=\sum_{i=1}^{7} \frac{\lambda_{i}}{3}\left(2\left\langle J_{i} X, Y\right\rangle J_{i} Z+\left\langle J_{i} Z, Y\right\rangle J_{i} X-\left\langle J_{i} Z, X\right\rangle J_{i} Y\right) .
$$

Some of the $\lambda_{i}$ 's can be equal, but up to relabelling, we can assume that $\lambda_{4} \neq \lambda_{5}, \lambda_{6} \neq \lambda_{7}$.

(b) $\nu=3, J_{1} J_{2}=J_{3}$, and the curvature tensor has the form

$$
\begin{aligned}
R(X, Y) Z & =\lambda_{0}(\langle X, Z\rangle Y-\langle Y, Z\rangle X) \\
& +\sum_{i=1}^{3} \frac{1}{3}\left(\lambda_{i}-\lambda_{0}\right)\left(2\left\langle J_{i} X, Y\right\rangle J_{i} Z+\left\langle J_{i} Z, Y\right\rangle J_{i} X-\left\langle J_{i} Z, X\right\rangle J_{i} Y\right) .
\end{aligned}
$$

Note that manifolds with curvature tensor (21) were studied in [7, Theorem 7.1], but our assumption is a little weaker: all the $\lambda_{i}$ 's can be nonconstant.

Proof. Let $\mathrm{Cl}(\nu)$ be a Clifford algebra on $\nu+1$ generators $1, x_{1}, \ldots, x_{\nu}$. Then the map $\rho: \operatorname{Cl}(\nu) \rightarrow \mathbb{R}^{n}$ defined on generators by $\rho(1)=I_{n}, \rho\left(x_{i}\right)=J_{i}$ is a representation of $\mathrm{Cl}(\nu)$ in $\mathbb{R}^{n}$.

From the representation theory of Clifford algebras $[1,8,9]$, we know that for $\nu=4,5,6$, there exists a unique (up to equivalency) irreducible representation $\rho_{\nu}$ of $\mathrm{Cl}(\nu)$ which is a representation in $\mathbb{R}^{8}$. The 
Clifford algebra $\mathrm{Cl}(7)$ has two inequivalent irreducible representations, both in $\mathbb{R}^{8}$. In fact, $J_{1} J_{2} \ldots J_{7}=$ $\rho\left(x_{1} x_{2} \ldots x_{7}\right)$ is always $\pm I_{8}$, and the choice of the sign determines one of two representations $\rho_{7}^{ \pm}$. Up to equivalency, the representations $\rho_{7}^{ \pm}$can be chosen in such a way that the operators $\tilde{J}_{i}=\rho_{7}^{ \pm}\left(x_{i}\right)$ are orthogonal and skew-symmetric. Similarly, $\mathrm{Cl}(3)$ has two inequivalent irreducible representations $\rho_{3}^{ \pm}$, both in $\mathbb{R}^{4}$, differing by the sign of $\rho_{3}^{ \pm}\left(x_{1} x_{2} x_{3}\right)= \pm I_{4}$.

First assume $\nu=7$. If $J_{1} \ldots J_{7}=-I_{8}$, replace $J_{7}$ by $-J_{7}$ (this does not change the form of the curvature tensor). As for any nonzero $X \in \mathbb{R}^{8}$, the vectors $X, J_{1} X, \ldots, J_{7} X$ form an orthogonal basis, with all the vectors of length $\|X\|$, we have $\|X\|^{2} Y-\langle X, Y\rangle X=\left\langle J_{i} X, Y\right\rangle J_{i} X$, and so by polarization $\langle X, Z\rangle Y-\langle Y, Z\rangle X=\sum_{i=1}^{7} \frac{1}{3}\left(2\left\langle J_{i} X, Y\right\rangle J_{i} Z+\left\langle J_{i} Z, Y\right\rangle J_{i} X-\left\langle J_{i} Z, X\right\rangle J_{i} Y\right)$. Substituting this to (19), we obtain $(20)$.

Let now $\nu=4,5,6$. Then $\rho_{\nu}$ is equivalent to a restriction of $\rho_{7}^{+}$to the subalgebra $\mathrm{Cl}(\nu) \subset \mathrm{Cl}(7)$ generated by $1, x_{1}, \ldots, x_{\nu}$. Denote $\tilde{J}_{i}=\rho_{7}^{+}\left(x_{i}\right)$ for $i=1, \ldots, 7$. Then there exist $T \in \operatorname{GL}(8)$ such that $J_{i}=T \tilde{J}_{i} T^{-1}$ for $i=1, \ldots, \nu$. As both $J_{i}$ and $\tilde{J}_{i}$ are skew-symmetric, we get $J_{i}\left(T T^{t}\right)=\left(T T^{t}\right) J_{i}$, and so every eigenspace of the symmetric operator $T T^{t}$ is an invariant subspace of $\rho_{\nu}$. Since $\rho_{\nu}$ is irreducible, $T T^{t}$ is proportional to the identity operator, and so we can assume that $T \in \mathrm{O}(8)$. Then for $i=\nu+1, \ldots, 7$, the operators $J_{i}=T \tilde{J}_{i} T^{-1}$ are skew-symmetric and orthogonal. The anticommutativity of $J_{1}, \ldots, J_{7}$ follows from that in $\mathrm{Cl}(7)$. Thus, the curvature tensor can be written in the form (19), with the summation ranging up to 7 , and with $\lambda_{i}=\lambda_{0}$ for $\nu<i \leq 7$. We then proceed as in the case $\nu=7$.

Finally, consider the case $\nu=3$. We have three inequivalent (reducible) representations of $\mathrm{Cl}(3)$ in $\mathbb{R}^{8}$ depending on the symmetric operator $S=J_{1} J_{2} J_{3}$ : either $S= \pm I_{8}$, or it has two orthogonal 4-dimensional eigenspaces $V_{ \pm}$corresponding to eigenvalues \pm 1 . The first case gives us the curvature tensor of the form (21) (up to switching $J_{3}$ to $-J_{3}$, if $S=I_{8}$ ). For the representation $\rho$ in the second case, consider the restriction of $\rho_{7}^{+}$to the subalgebra $\mathrm{Cl}(3) \subset \mathrm{Cl}(7)$ generated by $1, x_{1}, x_{2}, x_{3}$. Let $\tilde{J}_{i}=\rho_{7}^{+}\left(x_{i}\right), i=1,2,3$. We get a representation of $\mathrm{Cl}(3)$ in $\mathbb{R}^{8}$ which is equivalent to $\rho$, as $\tilde{J}_{1} \tilde{J}_{2} \tilde{J}_{3} \neq \pm I_{8}$. Then for some $T \in \mathrm{GL}(8), J_{i}=T \tilde{J}_{i} T^{-1}$, and so $J_{i}\left(T T^{t}\right)=\left(T T^{t}\right) J_{i}, i=1,2,3$. Hence the symmetric positively defined operator $T T^{t}$ has two eigenvalues, $\alpha_{ \pm}^{2}$, with eigenspaces $V_{ \pm}$, respectively. Define an operator $\tilde{T}$ by $\tilde{T}_{\mid V_{ \pm}}=\alpha_{ \pm}^{-1} T_{\mid V_{ \pm}}$. Then $\tilde{T}$ is orthogonal, and for $i=1,2,3, J_{i}=\tilde{T}_{i} \tilde{T}^{-1}$, as $V_{ \pm}$are the invariant subspaces of $J_{1}, J_{2}, J_{3}$. We then define $J_{i}, 4 \leq i \leq 7$ by $J_{i}=\tilde{T} \rho_{7}^{+}\left(x_{i}\right) \tilde{T}^{-1}$ and proceed as in the case $\nu=4,5,6$.

The eigenvalues of the Jacobi operator of the curvature tensor (20) are $\lambda_{1}, \ldots, \lambda_{7}$. As at least three of them are pairwise nonequal, they can be relabelled to satisfy $\lambda_{4} \neq \lambda_{5}, \lambda_{6} \neq \lambda_{7}$ (this can be done by an even permutation, not to violate the condition $J_{1} \ldots J_{7}=I_{8}$ ).

We are going to show that in each of the cases (a) and (b) of Lemma 5 , the manifold $M^{8}$ is rank-one symmetric.

\subsection{Case (a)}

In this case, the manifold carries a Hermitian almost octonion structure defined by the operators $J_{1}, \ldots, J_{7}$. The $\mathrm{Cl}(7)$-module $\mathbb{R}^{8}$ for the representation $\rho_{7}^{+}$can be described in terms of the octonion algebra $\mathbb{O}$ as follows. Let $1, e_{1}, \ldots, e_{7}$ be a fixed canonical basis of generators for $\mathbb{O}$, so that $e_{i}^{2}=-1, e_{i} e_{j}+e_{j} e_{i}=0, i \neq j$ (we will use the canonical basis with multiplication table given in [2, Ch. 3G]). Then there exists an orthogonal map $\psi: \mathbb{R}^{8} \rightarrow \mathbb{O}$ such that for every $i=1, \ldots, 7$ and every $X \in \mathbb{R}^{8}, \psi\left(J_{i} X\right)=\psi(X) e_{i}$.

Remark. Explicitly, one can construct $\psi$ from the given $J_{i}$ 's as follows: consider the set $S$ of triples $(i, j, k)$ with $e_{i} e_{j}=e_{k}$, according to the multiplication table. Any two triples from $S$ either contain exactly one element in common, or can be obtained from one another by a cyclic permutation. Then the operators $\left\{J_{i} J_{j} J_{k},(i, j, k) \in S\right\}$ in $\mathbb{R}^{8}$ are orthogonal, symmetric and pairwise commute, hence they 
have the same set of eigenvectors. One can show that it is possible to chose a common eigenvector $X^{0}$ whose eigenvalue is +1 for all the above operators. Then $\psi$ is defined by $\psi\left(X^{0}\right)=1, \psi\left(J_{i} X^{0}\right)=e_{i}$.

With some abuse of language, we identify, by $\psi$, vectors from $\mathbb{R}^{8}$ with octonions, so that a vector $X \in \mathbb{R}^{8}$ with coordinates $\left(X_{0}, X_{1}, \ldots, X_{7}\right)$ is the same as the octonion $X_{0} 1+X_{1} e_{1}+\ldots+X_{7} e_{7}$. It is convenient to introduce the following notation:

$$
J_{u} X:=\sum_{i=1}^{7} u_{i} J_{i} X=X u,
$$

for $X \in \mathbb{R}^{8}=\mathbb{O}, u=\sum_{i=1}^{7} u_{i} e_{i} \in \mathbb{O}^{\prime}:=1^{\perp}$. We will also abbreviate $J_{e_{i}}$ to $J_{i}$.

Throughout this Section we will use, without explicitly referring, the following identities (see [3, Sec. 2] for details):

$$
\begin{gathered}
a^{*}:=2\langle a, 1\rangle 1-a, \quad(a b)^{*}=b^{*} a^{*}, \quad a^{* *}=a, \quad a a^{*}=\|a\|^{2} 1, \quad\|a b\|=\|a\|\|b\|, \\
\left\langle a^{*}, b^{*}\right\rangle=\langle a, b\rangle, \quad\langle a, b c\rangle=\left\langle b^{*} a, c\right\rangle=\left\langle a c^{*}, b\right\rangle, \\
a(a b)=\left(a^{2}\right) b, \quad(b a) a=b\left(a^{2}\right), \quad a\left(a^{*} b\right)=(b a) a^{*}=\|a\|^{2} b \\
\left(a b^{*}\right) c+\left(a c^{*}\right) b=2\langle b, c\rangle a, \quad a\left(b^{*} c\right)+b\left(a^{*} c\right)=2\langle a, b\rangle c
\end{gathered}
$$

for any $a, b, c \in \mathbb{O}$. Note that $1^{*}=1, e_{i}^{*}=-e_{i}$. The algebra $\mathbb{O}$ is a division algebra, in particular, any nonzero octonion is invertible, with $a^{-1}=\|a\|^{-2} a^{*}$.

We will also use bioctonions $\mathbb{O} \otimes \mathbb{C}$, the algebra over the $\mathbb{C}$ with the same canonical basis of generator as $\mathbb{O}$. As all the above identities are polynomial, they still hold for bioctonions, with Euclidean scalar product in $\mathbb{C}^{8}$, the underlying linear space of $\mathbb{O} \otimes \mathbb{C}$. However, bioctonion algebra is no longer a division algebra (for example, because it has zero-divisors: $\left.\left(i 1+e_{1}\right)\left(i 1-e_{1}\right)=0\right)$.

The following Lemma will be in use later in the proof.

Lemma 6. Let $e \perp 1$ be a unit octonion, and let $L, F: \mathbb{O} \rightarrow \mathbb{O}$ be (R-)linear operators satisfying

$$
(Y e) L(Y)=Y F(Y) \quad \text { for all } Y \in \mathbb{O} \text {. }
$$

Then there exist $a, b, p \in \mathbb{O}$ such that

$$
L(Y)=\langle a, Y\rangle 1+\langle b, Y\rangle e+Y^{*} p .
$$

Proof. The set of linear operators $L$ for which there exists $F$ such that $L, F$ satisfy (22) is a linear subspace $\mathcal{S} \subset \operatorname{End}\left(\mathbb{R}^{8}\right)$. For any $p \in \mathbb{O}$, an operator $L(Y)=Y^{*} p$ is in $\mathcal{S}$, as $(Y e)\left(Y^{*} p\right)=2\langle Y e, Y\rangle p-$ $Y\left((Y e)^{*} p\right)=Y\left(-(Y e)^{*} p\right)$, and we can take $F(Y)=-(Y e)^{*} p$.

So, given an operator $L$ satisfying $(22)$ with some $F$, we can replace it by an operator $\hat{L}$ defined as $\hat{L}(Y)=L(Y)-Y^{*} L(1)$, still satisfying $(22)$ and having the property $\hat{L}(1)=0$. The operator $\hat{F}$ corresponding to $\hat{L}$ by (22) must also vanish at $Y=1$.

Denote $\mathcal{L}=(\operatorname{Span}(1, e))^{\perp}$. Let $u \in \mathcal{L}$ be a nonzero octonion and $t$ a real number. Substituting $Y=t 1+u$ in $(22)$ we get: $(t e+u e) \hat{L}(u)=(t 1+u) \hat{F}(u)$, so $\hat{F}(u)=e \hat{L}(u)$ and $(u e) \hat{L}(u)=u \hat{F}(u)$, hence

$$
(u e) \hat{L}(u)=u(e \hat{L}(u)) \text {. }
$$

It follows that for all $u \in \mathcal{L}, u \neq 0$, the octonion $\hat{L}(u)$ lies in an associative subalgebra of $\mathbb{O}$ generated by $u, e$. This subalgebra is $\operatorname{Span}(1, e, u, u e)$ and is isomorphic to $\mathbb{H}$. Then for some functions $\alpha, \beta, \gamma, \delta$ : $\mathcal{L} \rightarrow \mathbb{R}$ we have

$$
\hat{L}(u)=\alpha(u) 1+\beta(u) e+\gamma(u) u+\delta(u) u e .
$$

The functions $\alpha(u)=\langle\hat{L}(u), 1\rangle$ and $\beta(u)=\langle\hat{L}(u), e\rangle$ are linear, so there exist octonions $\hat{a}, \hat{b}$ such that $\alpha(u)=\langle\hat{a}, u\rangle, \beta(u)=\langle\hat{b}, u\rangle$. We want to show that $\gamma$ and $\delta$ are constants. Indeed, from (23) we 
get $\tilde{L}(u)=\gamma(u) u+\delta(u) u e$, where $\tilde{L}$ is a linear operator defined by $\tilde{L}(u)=\hat{L}(u)-\langle\hat{a}, u\rangle 1-\langle\hat{b}, u\rangle$ e. Then $\gamma(u)=\|u\|^{-2} C(u), \delta(u)=\|u\|^{-2} D(u)$, where $C(u)=\langle\tilde{L}(u), u\rangle, D(u)=\langle\tilde{L}(u), u e\rangle$ are quadratic forms on $\mathcal{L}$, and $\|u\|^{2} \tilde{L}(u)=C(u) u+D(u)$ ue. Taking the squared norm of the both sides we obtain $\|u\|^{2}\|\tilde{L}(u)\|^{2}=C^{2}(u)+D^{2}(u)$. As the sum of squares of polynomials $C, D$ in six real variables is divisible by $\|u\|^{2}$, each of them must be divisible by $\|u\|^{2}$ (see the Remark in Subsection 4.2). So $\gamma=\|u\|^{-2} C(u)$ and $\delta=\|u\|^{-2} D(u)$ are constants. Back to (23), we get

$$
\hat{L}(u)=\langle\hat{a}, u\rangle 1+\langle\hat{b}, u\rangle e+\gamma u+\delta u e .
$$

Then for an arbitrary $Y \in \mathbb{O}$,

$$
\begin{aligned}
\hat{L}(Y) & =\hat{L}(\langle Y, 1\rangle 1+\langle Y, e\rangle e+(Y-\langle Y, 1\rangle 1-\langle Y, e\rangle e)) \\
& =\langle Y, e\rangle \hat{L}(e)+\langle\hat{a}, Y-\langle Y, 1\rangle 1-\langle Y, e\rangle e\rangle 1+\langle\hat{b}, Y-\langle Y, 1\rangle 1-\langle Y, e\rangle e\rangle e \\
& +\gamma(Y-\langle Y, 1\rangle 1-\langle Y, e\rangle e)+\delta(Y-\langle Y, 1\rangle 1-\langle Y, e\rangle e) e \\
& =\langle Y, e\rangle \hat{L}(e)+\langle a, Y\rangle 1+\langle b, Y\rangle e+Y^{*} p,
\end{aligned}
$$

where $a=\hat{a}-\langle\hat{a}, 1\rangle 1-\langle\hat{a}, e\rangle e+\gamma 1+\delta e, b=\hat{b}-\langle\hat{b}, 1\rangle 1-\langle\hat{b}, e\rangle e-\gamma e+\delta 1, p=-\gamma 1-\delta e$. To finish the proof, it remains to show that the octonion $\hat{L}(e)$ lies in $\operatorname{Span}(1, e)$. To see that, substitute the above expression for $\hat{L}(Y)$ to $(22)$. Then

$$
\langle Y, e\rangle(Y e) \hat{L}(e)=Y \tilde{F}(Y),
$$

with a linear operator $\tilde{F}(Y)=\hat{F}(Y)-\left(\langle a, Y\rangle e-\langle b, Y\rangle 1-(Y e)^{*} p\right)$. Multiplying the above equation by $Y^{*}$ from the left we get $\langle Y, e\rangle Y^{*}((Y e) \hat{L}(e))=\|Y\|^{2} \tilde{F}(Y)$, so the octonion $Y^{*}((Y e) \hat{L}(e))$ viewed as an 8-dimensional vector, polynomial in $Y$, is divisible by $\|Y\|^{2}$. Then there exists an octonion $f$ such that $Y^{*}((Y e) \hat{L}(e))=\|Y\|^{2} f$ which implies $(Y e) \hat{L}(e)=Y f$, for all $Y$. If $Y=1$, this gives $f=e \hat{L}(e)$, and so $(Y e) \hat{L}(e)=Y(e \hat{L}(e))$. Therefore $\hat{L}(e)$ belongs to all associative subalgebras of $\mathbb{O}$ generated by $e, Y$, with $Y$ an arbitrary octonion. This is only possible when $\hat{L}(e) \in \operatorname{Span}(1, e)$.

In the next Lemma, we calculate covariant derivatives of the operators $J_{i}$, for later use in the second Bianchi identity.

Lemma 7. There exist linear functionals $A_{i}, B_{i j}$ on $\mathbb{R}^{8}, i, j=1, \ldots, 7$, with $B_{i j}=-B_{j i}$ such that for all $U \in \mathbb{R}^{8}$

$$
\nabla_{U} J_{i}=\sum_{j \neq i} B_{i j}(U) J_{j}+\sum_{j \neq i} A_{j}(U) J_{i} J_{j}=\sum_{j} B_{i j}(U) J_{j}+J_{i} J_{A(U)}+A_{i}(U) I_{8}
$$

where $A: \mathbb{O} \rightarrow \mathbb{O}^{\prime}\left(=\mathbb{O} \cap 1^{\perp}\right)$ is a linear operator defined by $A(U)=\sum_{j} A_{j}(U) e_{j}$.

Proof. The operator $\nabla_{U} J_{i}$ depends linearly on $U$ and is skew-symmetric. As the kernel of $\rho_{7}^{+}$is the ideal of $\mathrm{Cl}(7)$ generated by $x_{1} \ldots x_{7}-1$, the 28 skew-symmetric operators $\left\{J_{j}, J_{j} J_{k}\right\}, 1 \leq j<k \leq 7$ are linearly independent. In fact, they form a basis for $\mathfrak{o}(8)$, the space of skew-symmetric operators in $\mathbb{R}^{8}$, as $\operatorname{dim} \mathfrak{o}(8)=28$. So $\nabla_{U} J_{i}=\sum_{j} B_{i j}(U) J_{j}+\sum_{k, j} C_{i ; k j}(U) J_{k} J_{j}$ for some linear functionals $C_{i ; k j}, B_{i j}$, with $C_{i ; k j}=-C_{i ; j k}$.

As $\nabla_{U}\left(J_{i}^{2}\right)=0$, the operator $J_{i} \nabla_{U} J_{i}$ is skew-symmetric, so its symmetric part $-B_{i i}(U) I_{8}+$ $\sum_{k, j \neq i} C_{i ; k j}(U) J_{i} J_{k} J_{j}$ vanishes. Acting by $J_{i}$ from the left, we get a linear combination of $J_{i}, J_{k} J_{j}$ which is zero, therefore for all $i$ and for all $j, k \neq i, \quad B_{i i}=C_{i ; k j}=0$ which gives $\nabla_{U} J_{i}=\sum_{j \neq i} B_{i j}(U) J_{j}+$ $\sum_{j \neq i} C_{i ; j}(U) J_{i} J_{j}$, where $C_{i ; j}=2 C_{i ; i j}$

The equation $\nabla_{U}\left(J_{i} J_{s}+J_{s} J_{i}\right)=0$ implies that the operator $J_{i} \nabla_{U} J_{s}+J_{s} \nabla_{U} J_{i}=\sum_{j \neq s} B_{s j}(U) J_{i} J_{j}+$ $\sum_{j \neq s} C_{s ; j}(U) J_{i} J_{s} J_{j}+\sum_{j \neq i} B_{i j}(U) J_{s} J_{j}+\sum_{j \neq i} C_{i ; j}(U) J_{s} J_{i} J_{j}$ is skew-symmetric, for all $s \neq i$. Extracting the symmetric part we get: $-\left(B_{s i}(U)+B_{i s}(U)\right) I_{8}+\sum_{j \neq i, s}\left(C_{s ; j}(U)-C_{i ; j}(U)\right) J_{i} J_{s} J_{j}=0$. So $B_{s i}(U)+B_{i s}(U)=0$ and $C_{i ; j}(U)$ depends only on $j$ for $j \neq i$. Denoting $A_{i}(U)=C_{i ; j}(U), j \neq i$ we obtain (24). 
We will use the second Bianchi identity

$$
\left(\nabla_{U} R\right)(X, Y, Y, X)+\left(\nabla_{X} R\right)(Y, U, Y, X)+\left(\nabla_{Y} R\right)(U, X, Y, X)=0 .
$$

From (20), $R(V, Z) V=\sum_{i=1}^{7} \lambda_{i}\left\langle J_{i} V, Z\right\rangle J_{i} V$, and so for any three vectors $V, Z, W$,

$$
\begin{aligned}
\left(\nabla_{W} R\right)(V, Z) V & =\sum_{i=1}^{7} W\left(\lambda_{i}\right)\left\langle J_{i} V, Z\right\rangle J_{i} V \\
& +\sum_{i=1}^{7} \lambda_{i}\left(\left\langle\left(\nabla_{W} J_{i}\right) V, Z\right\rangle J_{i} V+\left\langle J_{i} V, Z\right\rangle\left(\nabla_{W} J_{i}\right) V\right) .
\end{aligned}
$$

Substituting this to $(25)$ we obtain

$$
\begin{aligned}
& \sum_{i=1}^{7}\left(X\left(\lambda_{i}\right)\left\langle J_{i} Y, U\right\rangle\left\langle J_{i} Y, X\right\rangle+Y\left(\lambda_{i}\right)\left\langle J_{i} U, X\right\rangle\left\langle J_{i} Y, X\right\rangle-U\left(\lambda_{i}\right)\left\langle J_{i} Y, X\right\rangle^{2}\right) \\
+ & \sum_{i=1}^{7} \lambda_{i}\left\langle J_{i} Y, X\right\rangle\left(2\left\langle\left(\nabla_{U} J_{i}\right) X, Y\right\rangle+\left\langle\left(\nabla_{X} J_{i}\right) Y, U\right\rangle+\left\langle\left(\nabla_{Y} J_{i}\right) U, X\right\rangle\right) \\
- & \sum_{i=1}^{7} \lambda_{i}\left(\left\langle J_{i} Y, U\right\rangle\left\langle\left(\nabla_{X} J_{i}\right) X, Y\right\rangle+\left\langle J_{i} X, U\right\rangle\left\langle\left(\nabla_{Y} J_{i}\right) Y, X\right\rangle\right)=0 .
\end{aligned}
$$

Lemma 8. The linear operator A introduced in Lemma 7 has the form

$$
A(U)=U^{*} m-\langle U, m\rangle 1, \quad \text { for some } m \in \mathbb{O} .
$$

Proof. The equation (27) is a polynomial equation in 24 real variables, the coordinates of the vectors $X, Y, U$. It must still hold if we allow $X, Y, U$ to be complex and extend the operators $J_{i}$ to $\mathbb{C}^{8}$ by complex linearity. Let $Y \in \mathbb{C}^{8}$ be a nonzero isotropic vector (bioctonion): $\|Y\|^{2}=0$. Denote $\mathcal{J} Y=$ $Y(\mathbb{O} \otimes \mathbb{C})=\operatorname{Span}_{\mathbb{C}}\left(J_{1} Y, \ldots, J_{7} Y\right)$. The space $\mathcal{J} Y$ is isotropic: the scalar product of any two vectors from $\mathcal{J} Y$ is zero. Choosing bioctonions $X, U \in \mathcal{J} Y$, we get $\left\langle J_{i} Y, X\right\rangle=\left\langle J_{i} Y, U\right\rangle=0$, for all $i=$ $1, \ldots 7$. Substituting this to (27) we obtain $\sum_{i=1}^{7} \lambda_{i}\left\langle\left(\nabla_{Y} J_{i}\right) Y, X\right\rangle\left\langle J_{i} X, U\right\rangle=0$ which simplifies to $\sum_{i=1}^{7} \lambda_{i}\left\langle J_{i} J_{A(Y)} Y, X\right\rangle\left\langle J_{i} X, U\right\rangle=0$ by (24). So $J_{A(Y)} Y \perp \sum_{i=1}^{7} \lambda_{i}\left\langle J_{i} X, U\right\rangle J_{i} X$ for all $X, U \in \mathcal{J} Y$. From this and the fact that $J_{A(Y)} Y \in \mathcal{J} Y \subset(\mathcal{J} Y)^{\perp}$ we get:

$$
J_{A(Y)} Y \perp \mathcal{L} Y:=\mathcal{J} Y+\operatorname{Span}_{\mathbb{C}}\left\{\sum_{i=1}^{7} \lambda_{i}\left\langle J_{i} X, U\right\rangle J_{i} X, \mid X, U \in \mathcal{J} Y\right\}
$$

The linear space $\mathcal{L} Y$ is at most 7-dimensional, as $Y \perp \mathcal{L} Y$. If we can produce at least one isotropic vector $Y$ such that $\operatorname{dim} \mathcal{L} Y=7$, then it will follow from (29) that for an open set of $Y$ 's on the isotropic cone $\|Y\|^{2}=0$, the vector $J_{A(Y)} Y$ is parallel to $Y$. As $J_{A(Y)} Y$ depends algebraically (in fact, quadratically) of $Y$, we will have $J_{A(Y)} Y \| Y$ for all isotropic vectors $Y$.

Take $Y=i 1+e_{1}$. Using the multiplication table of $[2, \mathrm{Ch} .3 \mathrm{G}]$ we find

$$
\mathcal{J}\left(i 1+e_{1}\right)=\operatorname{Span}_{\mathbb{C}}\left(i 1+e_{1}, i e_{2}+e_{3}, i e_{4}+e_{5}, i e_{6}-e_{7}\right) .
$$

Evaluating the expression $\sum_{i=1}^{7} \lambda_{i}\left\langle J_{i} X, U\right\rangle J_{i} X$ with $X=i e_{2}+e_{3}, U=i e_{6}-e_{7}$, with $X=i e_{2}+e_{3}, U=$ $i e_{4}+e_{5}$, and with $X=i e_{4}+e_{5}, U=i e_{2}+e_{3}$, we get, respectively, $-2\left(\lambda_{4}-\lambda_{5}\right)\left(i e_{6}+e_{7}\right), 2\left(\lambda_{6}-\right.$ $\left.\lambda_{7}\right)\left(-i e_{4}+e_{5}\right),-2\left(\lambda_{6}-\lambda_{7}\right)\left(i e_{2}-e_{3}\right)$. As $\lambda_{4} \neq \lambda_{5}, \lambda_{6} \neq \lambda_{7}$ by the assumption (Lemma 5$)$, the vectors $i e_{6}+e_{7},-i e_{4}+e_{5}, i e_{2}-e_{3}$ lie in $\mathcal{L}\left(i 1+e_{1}\right)$. It follows that

$$
\mathcal{L}\left(i 1+e_{1}\right) \supset \operatorname{Span}_{\mathbb{C}}\left\{i 1+e_{1}, e_{2}, e_{3}, e_{4}, e_{5}, e_{6}, e_{7}\right\},
$$

and so $\operatorname{dim} \mathcal{L}\left(i 1+e_{1}\right)=7$.

From the above, $J_{A(Y)} Y \| Y$ for all $Y$ with $\|Y\|^{2}=0$. So for all $i, j$, the coordinates of the vector $J_{A(Y)} Y$ viewed as complex polynomials of $Y_{0}, Y_{1}, \ldots, Y_{7}$ satisfy $\left(J_{A(Y)} Y\right)_{i} Y_{j}=\left(J_{A(Y)} Y\right)_{j} Y_{j}$ 
$\bmod \left(\|Y\|^{2}\right)$. As the ring $\mathbf{R} \otimes \mathbb{C}=\mathbb{C}\left[Y_{0}, \ldots, Y_{7}\right] /\left(\|Y\|^{2}\right)$ is a UFD (Fact 1, Subsection 4.2), there exists an element $r \in \mathbf{R} \otimes \mathbb{C}$ such that $\pi\left(\left(J_{A(Y)} Y\right)_{j}\right)=r \pi\left(Y_{j}\right)$ for all $j$, with $\pi: \mathbb{C}\left[Y_{0}, \ldots, Y_{7}\right] \rightarrow \mathbf{R} \otimes \mathbb{C}$ the natural projection. Lifting up, we find a polynomial function $f \in \pi^{-1}(r)$ and a polynomial vector $F$ such that $J_{A(Y)} Y=f(Y) Y+\|Y\|^{2} F(Y)$ for all $Y \in \mathbb{C}^{8}$. As $A(Y)$ is a linear operator, the left-hand side is a polynomial vector with all the components of degree 2 . As the polynomial $\|Y\|^{2}$ is irreducible, we can choose $f \in \pi^{-1}(r)$ to be linear, and $F$ to be a constant. So there exist $m, c \in \mathbb{O} \otimes \mathbb{C}$ such that for all $Y \in \mathbb{O} \otimes \mathbb{C}$,

$$
Y A(Y)\left(=J_{A(Y)} Y\right)=\langle c, Y\rangle Y+\|Y\|^{2} m .
$$

Multiplying by $Y^{*}$ from the left and dividing through by $\|Y\|^{2}$ we get $A(Y)=\langle c, Y\rangle 1+Y^{*} m$. Since $A(Y) \perp 1$, we must have $\langle c, Y\rangle+\langle m, Y\rangle=0$, and so $c=-m$. Also, as $A(Y)$ is real (lies in $\mathbb{O} \subset \mathbb{O} \otimes \mathbb{C}$ ), when $Y$ is real, $m$ has to be real, as well. So, for some $m \in \mathbb{O}$,

$$
A(Y)=-\langle m, Y\rangle 1+Y^{*} m
$$

Lemma 9. All the $\lambda_{k}$ 's are constant:

$$
\nabla \lambda_{k}=0, \quad \text { for all } k=1, \ldots, 7,
$$

and the operators $B_{i j}$ introduced in Lemma 7 satisfy

$$
\left(\lambda_{i}-\lambda_{j}\right) B_{i j}(Y)=-\left(\lambda_{i}-\lambda_{j}\right)\left\langle\left(m e_{j}\right) e_{i}, Y\right\rangle, \quad j \neq i
$$

Proof. Substitute $X=J_{k} Y=Y e_{k}$ in (27). We have $\left\langle J_{i} Y, X\right\rangle=\|Y\|^{2} \delta_{i k}$, and the second sum is nonzero only when $i=k$. Moreover, $\left\langle\left(\nabla_{U} J_{k}\right) X, Y\right\rangle=-\left\langle J_{k} Y,\left(\nabla_{U} J_{k}\right) Y\right\rangle=0$. Also, from (28), (24) we get:

$$
\begin{aligned}
\left\langle\left(\nabla_{X} J_{k}\right) Y, U\right\rangle+\left\langle\left(\nabla_{Y} J_{k}\right) U, X\right\rangle & =\sum_{j}\left(B_{k j}(X)\left\langle J_{j} Y, U\right\rangle-B_{k j}(Y)\left\langle J_{j} X, U\right\rangle\right) \\
& +\left\langle J_{k} J_{A(X)} Y+\left\langle A(X), e_{k}\right\rangle Y-J_{k} J_{A(Y)} X-\left\langle A(Y), e_{k}\right\rangle X, U\right\rangle \\
& =\sum_{j}\left(B_{k j}(X)\left\langle Y e_{j}, U\right\rangle-B_{k j}(Y)\left\langle X e_{j}, U\right\rangle\right) \\
& +\left\langle\left(Y\left(X^{*} m\right)-X\left(Y^{*} m\right)\right) e_{k}, U\right\rangle-2(\langle X, m\rangle\langle X, U\rangle+\langle Y, m\rangle\langle Y, U\rangle) .
\end{aligned}
$$

As $\left(Y\left(X^{*} m\right)-X\left(Y^{*} m\right)\right) e_{k}=2\left(Y\left(X^{*} m\right)\right) e_{k}=-2\left(Y\left(X^{*} m\right)\right) e_{k}^{*}=-4\left\langle X^{*} m, e_{k}\right\rangle Y+2 Y e_{k}\left(X^{*} m\right)^{*}=$ $-4\left\langle m, X e_{k}\right\rangle Y+2 X\left(-X^{*} m+2\left\langle X^{*} m, 1\right\rangle 1\right)=4(\langle Y, m\rangle Y+\langle X, m\rangle X)-2\|Y\|^{2} m$, we find

$$
\begin{aligned}
& \sum_{i} \lambda_{i}\left\langle J_{i} Y, X\right\rangle\left(2\left\langle\left(\nabla_{U} J_{i}\right) X, Y\right\rangle+\left\langle\left(\nabla_{X} J_{i}\right) Y, U\right\rangle+\left\langle\left(\nabla_{Y} J_{i}\right) U, X\right\rangle\right) \\
= & \lambda_{k}\|Y\|^{2}\left(\sum_{j}\left(B_{k j}(X)\left\langle Y e_{j}, U\right\rangle-B_{k j}(Y)\left\langle X e_{j}, U\right\rangle\right)-2\|Y\|^{2}\langle m, U\rangle+2(\langle X, m\rangle\langle X, U\rangle+\langle Y, m\rangle\langle Y, U\rangle)\right) .
\end{aligned}
$$

In a similar way, the third sum in (27) simplifies to

$$
\begin{array}{r}
-\|Y\|^{2} \sum_{i} \lambda_{i}\left(-B_{i k}(X)\left\langle Y e_{i}, U\right\rangle+B_{i k}(Y)\left\langle X e_{i}, U\right\rangle+\left\langle m e_{i}, Y\right\rangle\left\langle Y e_{i}, U\right\rangle+\left\langle m e_{i}, X\right\rangle\left\langle X e_{i}, U\right\rangle\right) \\
-\lambda_{k}\|Y\|^{2}(\langle X, m\rangle\langle X, U\rangle+\langle Y, m\rangle\langle Y, U\rangle) .
\end{array}
$$

Substituting all this to (27), dividing through by $\|Y\|^{2}$ and observing that the equation is linear with respect to $U$, we come to the following octonion equation:

$$
\begin{aligned}
X\left(\lambda_{k}\right) X+ & Y\left(\lambda_{k}\right) Y-\|Y\|^{2} \nabla \lambda_{k}-2 \lambda_{k}\|Y\|^{2} m+\lambda_{k}(\langle X, m\rangle X+\langle Y, m\rangle Y) \\
& -\sum_{i} \lambda_{i}\left(\left\langle m e_{i}, Y\right\rangle Y e_{i}+\left\langle m e_{i}, X\right\rangle X e_{i}\right)+\sum_{j}\left(\lambda_{k}-\lambda_{j}\right)\left(B_{k j}(X) Y e_{j}-B_{k j}(Y) X e_{j}\right)=0,
\end{aligned}
$$


where $X=Y e_{k}$. As $2\|Y\|^{2} m=\|Y\|^{2} m+\|X\|^{2} m=\sum_{i}\left\langle m, Y e_{i}\right\rangle Y e_{i}+\langle m, Y\rangle Y+\sum_{i}\left\langle m, X e_{i}\right\rangle X e_{i}+$ $\langle m, X\rangle X=-\sum_{i}\left(\left\langle m e_{i}, Y\right\rangle Y e_{i}+\left\langle m e_{i}, X\right\rangle X e_{i}\right)+\langle m, Y\rangle Y+\langle m, X\rangle X$, we get

$X\left(\lambda_{k}\right) X+Y\left(\lambda_{k}\right) Y-\|Y\|^{2} \nabla \lambda_{k}+\sum_{j}\left(\lambda_{k}-\lambda_{j}\right)\left(\left(B_{k j}(X)+\left\langle m e_{j}, Y\right\rangle\right) Y e_{j}+\left(-B_{k j}(Y)+\left\langle m e_{j}, X\right\rangle\right) X e_{j}\right)=0$.

As $\|Y\|^{2} \nabla \lambda_{k}=Y\left(Y^{*} \nabla \lambda_{k}\right)$ and $X=Y e_{k}$, the above equation can be written in the form

$$
\left(Y e_{k}\right) L_{k}(Y)=Y F_{k}(Y)
$$

where, for every $k=1, \ldots, 7, L_{k}$ and $F_{k}$ are linear operators in $\mathbb{O}$, with $L_{k}(Y) \perp 1$, given by

$$
\begin{aligned}
& L_{k}(Y)=\sum_{j}\left(\lambda_{k}-\lambda_{j}\right)\left(B_{k j}(Y)+\left\langle\left(m e_{j}\right) e_{k}, Y\right\rangle\right) e_{j}, \\
& F_{k}(Y)=\left(Y e_{k}\right)\left(\lambda_{k}\right) e_{k}+Y\left(\lambda_{k}\right) 1-Y^{*} \nabla \lambda_{k}+\sum_{j}\left(\lambda_{k}-\lambda_{j}\right)\left(B_{k j}\left(Y e_{k}\right)+\left\langle m e_{j}, Y\right\rangle\right) e_{j}
\end{aligned}
$$

Applying Lemma 6 , we find $L_{k}(Y)=\left\langle a_{k}, Y\right\rangle 1+\left\langle b_{k}, Y\right\rangle e_{k}+Y^{*} p_{k}$, for some $a_{k}, b_{k}, p_{k} \in \mathbb{O}$. Since $L_{k}(Y) \perp 1$, we get $a_{k}=-p_{k}$, and so

$$
L_{k}(Y)=-\left\langle p_{k}, Y\right\rangle 1+\left\langle b_{k}, Y\right\rangle e_{k}+Y^{*} p_{k}, \quad k=1, \ldots, 7 .
$$

From (32), (33) we obtain, for $j \neq k$ :

$$
\left\langle L_{k}(Y), e_{j}\right\rangle=\left(\lambda_{k}-\lambda_{j}\right)\left(B_{k j}(Y)+\left\langle\left(m e_{j}\right) e_{k}, Y\right\rangle\right)=-\left\langle p_{k} e_{j}, Y\right\rangle
$$

But the middle expression is symmetric with respect to $j, k$, as $B_{k j}=-B_{j k}$, so $p_{k} e_{j}=p_{j} e_{k}$, for all $j \neq k$. It follows that $p_{k} e_{k}=-p_{j} e_{j}$, hence all the $p_{k}$ 's vanish. Again, from $(32,33)$ it follows that $\left\langle L_{k}(Y), e_{k}\right\rangle=0=\left\langle b_{k}, Y\right\rangle$, so $b_{k}=0$, and therefore $L_{k}(Y)=0$. Then also $F_{k}(Y)=0$, and by (32) we obtain

$$
\begin{gathered}
\left(\lambda_{k}-\lambda_{j}\right) B_{k j}(Y)=-\left(\lambda_{k}-\lambda_{j}\right)\left\langle\left(m e_{j}\right) e_{k}, Y\right\rangle, \quad j \neq k \\
\left(Y e_{k}\right)\left(\lambda_{k}\right) e_{k}+Y\left(\lambda_{k}\right) 1-Y^{*} \nabla \lambda_{k}=0 .
\end{gathered}
$$

The former equation proves (31), the latter one, after multiplying by $Y$ from the left, implies that $\nabla \lambda_{k}$ lies in the two-dimensional space $\operatorname{Span}\left(Y, Y e_{k}\right)$, for any nonzero $Y \in \mathbb{O}$, and so $\nabla \lambda_{k}=0$ for all $k=1, \ldots, 7$.

It now follows from (26) and (30) that for any $X, Y$,

$$
\left(\nabla_{X} R\right)(X, Y) X=\sum_{i} \lambda_{i}\left(\left\langle\left(\nabla_{X} J_{i}\right) X, Y\right\rangle J_{i} X+\left\langle J_{i} X, Y\right\rangle\left(\nabla_{X} J_{i}\right) X\right) .
$$

As by $(24,28), \quad\left(\nabla_{X} J_{i}\right) X=\sum_{j} B_{i j}(X) J_{j} X+\|X\|^{2} J_{i} m-\langle X, m\rangle J_{i} X+\left\langle m, J_{i} X\right\rangle X$, we get

$$
\begin{aligned}
\left(\nabla_{X} R\right)(X, Y) X & =\sum_{i} \lambda_{i}\left(\left\langle\sum_{j} B_{i j}(X) J_{j} X+\|X\|^{2} J_{i} m-\langle X, m\rangle J_{i} X+\left\langle m, J_{i} X\right\rangle X, Y\right\rangle J_{i} X\right. \\
& \left.+\left\langle J_{i} X, Y\right\rangle\left(\sum_{j} B_{i j}(X) J_{j} X+\|X\|^{2} J_{i} m-\langle X, m\rangle J_{i} X+\left\langle m, J_{i} X\right\rangle X\right)\right) \\
& =\sum_{i j}\left(\lambda_{i}-\lambda_{j}\right) B_{i j}(X)\left\langle J_{j} X, Y\right\rangle J_{i} X-2\langle X, m\rangle \sum_{i} \lambda_{i}\left\langle J_{i} X, Y\right\rangle J_{i} X \\
& +\sum_{i} \lambda_{i}\left(\left\langle\|X\|^{2} J_{i} m+\left\langle m, J_{i} X\right\rangle X, Y\right\rangle J_{i} X+\left\langle J_{i} X, Y\right\rangle\left(\|X\|^{2} J_{i} m+\left\langle m, J_{i} X\right\rangle X\right)\right) .
\end{aligned}
$$


Now from (31)

$$
\begin{aligned}
& \sum_{i j}\left(\lambda_{i}-\lambda_{j}\right) B_{i j}(X)\left\langle J_{j} X, Y\right\rangle J_{i} X=-\sum_{i j}\left(\lambda_{i}-\lambda_{j}\right)\left\langle\left(m e_{j}\right) e_{i}, X\right\rangle\left\langle J_{j} X, Y\right\rangle J_{i} X \\
= & \sum_{i j} \lambda_{j}\left\langle\left(m e_{j}\right) e_{i}, X\right\rangle\left\langle J_{j} X, Y\right\rangle J_{i} X-\sum_{i j} \lambda_{i}\left\langle\left(m e_{j}\right) e_{i}, X\right\rangle\left\langle J_{j} X, Y\right\rangle J_{i} X \\
= & -\sum_{i}\left\langle\sum_{j} \lambda_{j}\left\langle J_{j} X, Y\right\rangle J_{j} m, J_{i} X\right\rangle J_{i} X \\
& -\sum_{i} \lambda_{i}\left(\sum_{j \neq i}\left\langle J_{i} m, J_{j} X\right\rangle\left\langle J_{j} X, Y\right\rangle-\langle m, X\rangle\left\langle J_{i} X, Y\right\rangle\right) J_{i} X .
\end{aligned}
$$

As $\sum_{i}\left\langle U, J_{i} X\right\rangle J_{i} X=\|X\|^{2} U-\langle U, X\rangle X, \sum_{i}\left\langle U, J_{i} X\right\rangle\left\langle V, J_{i} X\right\rangle=\|X\|^{2}\langle U, V\rangle-\langle U, X\rangle\langle V, X\rangle$, for any $U, V$, the above expression simplifies to

$$
\begin{array}{r}
\sum_{i j}\left(\lambda_{i}-\lambda_{j}\right) B_{i j}(X)\left\langle J_{j} X, Y\right\rangle J_{i} X=-\|X\|^{2} \sum_{j} \lambda_{j}\left\langle J_{j} X, Y\right\rangle J_{j} m+\sum_{j} \lambda_{j}\left\langle J_{j} X, Y\right\rangle\left\langle J_{j} m, X\right\rangle X \\
-\sum_{i} \lambda_{i}\left(\|X\|^{2}\left\langle J_{i} m, Y\right\rangle-2\langle m, X\rangle\left\langle J_{i} X, Y\right\rangle-\left\langle J_{i} m, X\right\rangle\langle Y, X\rangle\right) J_{i} X .
\end{array}
$$

Substituting to (34) we obtain

$$
\left(\nabla_{X} R\right)(X, Y) X=0 \text {. }
$$

So $\nabla R=0$ and the manifold $M^{8}$ is locally symmetric. The fact that $M^{8}$ is rank-one symmetric follows from [7, Lemma 2.3].

\subsection{Case (b)}

In this case, the manifold carries an almost hypercomplex Hermitian structure defined by the operators $J_{1}, J_{2}, J_{3}$, with $J_{1} J_{2}=J_{3}$, and with the curvature tensor given by (21).

The second Bianchi identity $\left(\nabla_{U} R\right)(X, Y, Y, X)+\left(\nabla_{X} R\right)(Y, U, Y, X)+\left(\nabla_{Y} R\right)(U, X, Y, X)=0$ takes the form

$$
\begin{aligned}
& U\left(\lambda_{0}\right)\left(\langle X, Y\rangle^{2}-\|X\|^{2}\|Y\|^{2}\right) \\
+ & X\left(\lambda_{0}\right)\left(\|Y\|^{2}\langle U, X\rangle-\langle U, Y\rangle\langle X, Y\rangle\right)+Y\left(\lambda_{0}\right)\left(\|X\|^{2}\langle U, Y\rangle-\langle U, X\rangle\langle X, Y\rangle\right) \\
+ & \sum_{i=1}^{3}\left(X\left(\lambda_{i}-\lambda_{0}\right)\left\langle J_{i} Y, U\right\rangle\left\langle J_{i} Y, X\right\rangle+Y\left(\lambda_{i}-\lambda_{0}\right)\left\langle J_{i} U, X\right\rangle\left\langle J_{i} Y, X\right\rangle-U\left(\lambda_{i}-\lambda_{0}\right)\left\langle J_{i} Y, X\right\rangle^{2}\right) \\
+ & \sum_{i=1}^{3}\left(\lambda_{i}-\lambda_{0}\right)\left(\left(2\left\langle\left(\nabla_{U} J_{i}\right) X, Y\right\rangle+\left\langle\left(\nabla_{X} J_{i}\right) Y, U\right\rangle+\left\langle\left(\nabla_{Y} J_{i}\right) U, X\right\rangle\right)\left\langle J_{i} Y, X\right\rangle\right. \\
- & \left.\left\langle J_{i} Y, U\right\rangle\left\langle\left(\nabla_{X} J_{i}\right) X, Y\right\rangle-\left\langle J_{i} X, U\right\rangle\left\langle\left(\nabla_{Y} J_{i}\right) Y, X\right\rangle\right)=0 .
\end{aligned}
$$

For a nonzero vector $Y$, let $\mathcal{L} Y=\operatorname{Span}\left(Y, J_{1} Y, J_{2} Y, J_{3} Y\right)$. Note that $\mathcal{L}\left(J_{i} Y\right)=\mathcal{L} Y$, and that if $X \perp \mathcal{L} Y$, then also $\mathcal{L} X \perp \mathcal{L} Y$.

Taking $U, X \perp \mathcal{L} Y$, with $J_{1} X=U$, in (35) we obtain

$$
\|Y\|^{2} U\left(\lambda_{0}\right)-\left(\lambda_{1}-\lambda_{0}\right)\left\langle\left(\nabla_{Y} J_{1}\right) Y, J_{1} U\right\rangle=0 \text { for all } U, Y \text { with } U \perp \mathcal{L} Y .
$$

On the other hand, taking $X=J_{1} Y, U \perp \mathcal{L} Y=\mathcal{L} X$ in (35) gives

$$
\|Y\|^{2} U\left(\lambda_{1}\right)-\left(\lambda_{1}-\lambda_{0}\right)\left(2\left\langle\left(\nabla_{U} J_{1}\right) X, Y\right\rangle+\left\langle\left(\nabla_{X} J_{1}\right) Y, U\right\rangle+\left\langle\left(\nabla_{Y} J_{1}\right) U, X\right\rangle\right)=0 .
$$

Using (36) and the fact that the operator $J_{1}$ is orthogonal and skew-symmetric we get:

$\left\langle\left(\nabla_{U} J_{1}\right) X, Y\right\rangle=-\left\langle\left(\nabla_{U} J_{1}\right) Y, J_{1} Y\right\rangle=0$,

$\left(\lambda_{1}-\lambda_{0}\right)\left\langle\left(\nabla_{Y} J_{1}\right) U, X\right\rangle=-\left(\lambda_{1}-\lambda_{0}\right)\left\langle U,\left(\nabla_{Y} J_{1}\right) J_{1} Y\right\rangle=-\left(\lambda_{1}-\lambda_{0}\right)\left\langle J_{1} U,\left(\nabla_{Y} J_{1}\right) Y\right\rangle=-\|Y\|^{2} U\left(\lambda_{0}\right)$,

$\left(\lambda_{1}-\lambda_{0}\right)\left\langle\left(\nabla_{X} J_{1}\right) Y, U\right\rangle=-\left(\lambda_{1}-\lambda_{0}\right)\left\langle U,\left(\nabla_{X} J_{1}\right) J_{1} X\right\rangle=-\|X\|^{2} U\left(\lambda_{0}\right)=-\|Y\|^{2} U\left(\lambda_{0}\right)$, 
so $(37)$ takes the form $U\left(\lambda_{1}+2 \lambda_{0}\right)=0$. Then $\lambda_{1}+2 \lambda_{0}=c_{1}$, a constant. Similarly, $\lambda_{2}+2 \lambda_{0}=c_{2}$, $\lambda_{3}+2 \lambda_{0}=c_{3}$ for some constants $c_{2}, c_{3}$.

The manifold $M^{8}$ is pointwise Osserman, hence Einstein, so $\lambda_{1}+\lambda_{2}+\lambda_{3}+4 \lambda_{0}=C$, a constant (the scalar curvature). Then $\lambda_{0}=\left(c_{1}+c_{2}+c_{3}-C\right) / 2$ is constant, and the claim follows from [7, Theorem 7.1].

Remark. The above computation similarly works for 4-dimensional pointwise Osserman manifolds with Cliff(1)-structure, including "generalized complex space forms" [15]. However, the equation $\lambda_{1}+2 \lambda_{0}=$ const gives nothing new: it is just the condition of constancy of the scalar curvature.

\section{References}

[1] M.F.Atiah, R.Bott, A.Shapiro, Clifford modules, Topology, 3, suppl.1 (1964), $3-38$.

[2] A.L.Besse, Manifolds all of whose geodesics are closed, Ergebnisse der Mathematik und ihrer Grenzgebiete, 93(1978), Springer-Verlag.

[3] R.Brown, A.Gray, Riemannian manifolds with holonomy group $\operatorname{Spin}(9)$, Diff. Geom. in honor of K.Yano, Kinokuniya, Tokyo (1972), $41-59$.

[4] Q.-S.Chi, A curvature characterization of certain locally rank-one symmetric spaces, J. Differ. Geom., 28(1988), $187-202$.

[5] E.García-Río, D.N. Kupeli, R.Vázguez-Lorenzo, Osserman manifolds in Semi-Riemannian Geometry, Lecture Notes in Mathematics, 1777(2002), Springer-Verlag.

[6] P.Gilkey, Manifolds whose curvature operator has constant eigenvalues at the basepoint, J. Geom. Anal., 4(1994), 155 - 158.

[7] P.Gilkey, A.Swann, L.Vanhecke, Isoperimetric geodesic spheres and a conjecture of Osserman concerning the Jacobi operator, Quart. J. Math. Oxford (2), 46(1995), 299 - 320.

[8] D.Husemoller, Fiber bundles, (1975), Springer-Verlag.

[9] H.B.Lawson, M.-L.Michelsohn, Spin geometry, (1989), Princeton Univ. Press.

[10] J.Levine, Imbedding and immersion of real projective spaces, Proc. Amer. Math. Soc., 14 (1963), $801-803$.

[11] M.Nagata, A remark on the unique factorization theorem, J. Math. Soc. Japan, 9, (1957), 143-145.

[12] Y.Nikolayevsky, Osserman manifolds and Clifford structures, Houston J. Math., 29(2003), 59-75.

[13] Y.Nikolayevsky, Two theorems on Osserman manifolds, Diff. Geom. Appl., 18(2003), 239 - 253.

[14] Y. Nikolayevsky, Osserman Conjecture in dimension $n \neq 8,16$, preprint, http://au.arxiv.org/abs/math.DG/0204258

[15] Z.Olszak, On the existence of generalized space forms, Israel J. Math., 65(1989), $214-218$.

[16] R.Osserman, Curvature in the eighties, Amer. Math. Monthly, 97(1990), 731 - 756.

[17] A.Pfister, Quadratic forms with applications to algebraic geometry and topology, London Math. Soc. Lecture Notes Ser., 217, (1995), Cambridge Univ. Press. 